\title{
1 Genomic and transcriptomic analyses of the \\ 2 subterranean termite Reticulitermes speratus: gene 3 duplication facilitates social evolution
}

5 Shuji Shigenobu ${ }^{*+1,2}$, Yoshinobu Hayashi $^{+3}$, Dai Watanabe ${ }^{4,5}$, Gaku Tokuda ${ }^{6}$, 6 Masaru Y Hojo ${ }^{6,19}$, Kouhei Toga ${ }^{5,7}$, Ryota Saiki ${ }^{5}$, Hajime Yaguchi ${ }^{5,8}$, Yudai

7 Masuoka $^{5,9}$, Ryutaro Suzuki $^{5,10}$, Shogo Suzuki ${ }^{5}$, Moe Kimura ${ }^{11}$, Masatoshi

8 Matsunami $^{4,12}$, Yasuhiro Sugime ${ }^{4}$, Kohei Oguchi ${ }^{4,10,13}$, Teruyuki Niimi ${ }^{2,14}$, Hiroki

9 Gotoh $^{15,20}$, Masaru K Hojo ${ }^{8}$, Satoshi Miyazaki ${ }^{16}$, Atsushi Toyoda ${ }^{17}$, Toru Miura*4,13,

10 Kiyoto Maekawa*18

12 1) NIBB Research Core Facilities, National Institute for Basic Biology, Okazaki,

13 444-8585 Japan

14 2) Department of Basic Biology, School of Life Science, The Graduate University for

15 Advanced Studies, SOKENDAI, Nishigonaka 38, Myodaiji, Okazaki, Aichi 444-

16 8585, Japan

17 3) Department of Biology, Keio University, Hiyoshi, Yokohama, 223-8521, Japan

18 4) Faculty of Environmental Earth Science, Hokkaido University, Sapporo,

19 Hokkaido, 060-0810, Japan

20 5) Graduate School of Science and Engineering, University of Toyama, Toyama,

21 930-8555, Japan

22 6) Tropical Biosphere Research Center, COMB, University of the Ryukyus,

23 Nishihara, Okinawa 903-0213, Japan

24 7) Department of Biosciences, College of Humanities and Sciences, Nihon

25 University, Setagaya-ku, Tokyo, 156-8550, Japan

26 8) Department of Bioscience, School of Science and Technology, Kwansei Gakuin

27 University, Sanda, Hyogo, 669-1337, Japan

28 9) Institute of Agrobiological Sciences, NARO (National Agriculture and Food

29 Research Organization), Tsukuba, Japan

30 10) Bioproduction Research Institute, National Institute of Advanced Industrial

31 Science and Technology (AIST), Tsukuba, Japan

32 11) School of Science, University of Toyama, Toyama, 930-8555, Japan

33 12) Graduate School of Medicine, University of the Ryukyus, Nishihara, Okinawa

34 903-0125, Japan

35 13) Misaki Marine Biological Station, School of Science, The University of Tokyo,

36 Miura, Kanagawa, 238-0225, Japan 
37 14) Division of Evolutionary Developmental Biology, National Institute for Basic

38 Biology, Okazaki, 444-8585 Japan

39 15) Ecological Genetics Laboratory, Department of Genomics and Evolutionary

40 Biology, National Institute of Genetics, Mishima, Shizuoka, 411-8540, Japan

41 16) Graduate School of Agriculture, Tamagawa University, Machida, Tokyo 194-

42 8610, Japan

43 17) Advanced Genomics Center, National Institute of Genetics, Mishima, Shizuoka,

44 411-8540 Japan

45 18) Faculty of Science, Academic Assembly, University of Toyama, Gofuku,

46 Toyama, 930-8555, Japan

47 19) Global Education Institute, University of the Ryukyus, Nishihara, Okinawa 903-

48 0213, Japan

49 20) Department of Biological Sciences, Faculty of Science, Shizuoka University,

50 Shizuoka, Shizuoka 422-8529, Japan

51

52

$53{ }^{*}$ corresponding authors. Email: shige@nibb.ac.jp, miu@mmbs.s.u-tokyo.ac.jp,

54 kmaekawa@sci.u-toyama.ac.jp

55

$56+$ contributed equally

57 


\section{Summary}

59 Termites are model social organisms characterized by a polyphenic caste system.

60 Subterranean termites (Rhinotermitidae) are ecologically and economically

61 important species, including acting as destructive pests. Rhinotermitidae occupies

62 an important evolutionary position within the clade representing an intermediate

63 taxon between the higher (Termitidae) and lower (other families) termites. Here, we

64 report the genome, transcriptome and methylome of the Japanese subterranean

65 termite Reticulitermes speratus. The analyses highlight the significance of gene

66 duplication in social evolution in this termite. Gene duplication associated with

67 caste-biased gene expression is prevalent in the $R$. speratus genome. Such

68 duplicated genes encompass diverse categories related to social functions,

69 including lipocalins (chemical communication), cellulases (wood digestion and

70 social interaction), lysozymes (social immunity), geranylgeranyl diphosphate

71 synthase (social defense) and a novel class of termite lineage-specific genes with

72 unknown functions. Paralogous genes were often observed in tandem in the

73 genome, but the expression patterns were highly variable, exhibiting caste biases.

74 Some duplicated genes assayed were expressed in caste-specific organs, such as

75 the accessory glands of the queen ovary and frontal glands in soldier heads. We

76 propose that gene duplication facilitates social evolution through regulatory

77 diversification leading to caste-biased expression and subfunctionalization and/or

78 neofunctionalization that confers caste-specialized functions.

\section{Significance Statement}

81 Termites are model social organisms characterized by a sophisticated caste

82 system, where distinct castes arise from the same genome. Our genomics data of

83 Japanese subterranean termite provides insights into the evolution of the social

84 system, highlighting the significance of gene duplication. Gene duplication

85 associated with caste-biased gene expression is prevalent in the termite genome.

86 Many of the duplicated genes were related to social functions, such as chemical

87 communication, social immunity and defense, and they often expressed in caste-

88 specific organs. We propose that gene duplication facilitates social evolution 
bioRxiv preprint doi: https://doi.org/10.1101/2021.07.11.451559; this version posted July 12, 2021. The copyright holder for this preprint (which was not certified by peer review) is the author/funder. All rights reserved. No reuse allowed without permission.

89 through regulatory diversification leading to caste-biased expression and functional

90 specialization. In addition, since subterranean termites are ecologically and

91 economically important species including destructive pests in the world, our

92 genomics data serves as a foundation for these studies. 


\section{Introduction}

94 The evolution of eusociality, i.e., animal societies defined by the

95 reproductive division of labor, cooperative brood care and multiple overlapping

96 generations, represents one of the major transitions in evolution, having increased

97 the level of biological complexity (1). Eusocial insects such as bees, wasps, ants

98 and termites show sophisticated systems based on the division of labor among

99 castes, which is one of the pinnacles of eusocial evolution (2). Recent advances in

100 molecular biological technologies and omics studies have revealed many molecular

101 mechanisms underlying eusociality and have led to the establishment of a new field

102 of study known as "sociogenomics" (3). The genomes of major eusocial

103 hymenopteran lineages, i.e., ants, bees and wasps, have been sequenced, and the

104 differences in gene expression, DNA methylation (4-6)(7) (8-11) and histone

105 modification (12) (13) among castes have been explored. These sociogenomics

106 studies in hymenopterans revealed some genetic bases of social evolution,

107 including the co-option of genetic toolkits of conserved genes, changes in protein-

108 coding genes, cis-regulatory evolution leading to genetic network reconstruction,

109 epigenetic modifications and taxonomically restricted genes (TRG) $(14,15)$.

Isoptera (termites) is another representative insect lineage exhibiting highly

112 sophisticated eusociality and a wide range of social complexities (16). Termites are

113 hemimetabolous and diploid insects that are phylogenetically distant from

114 hymenopterans with holometaboly and haplodiploidy. Termite societies are

115 characterized by reproductives of both sexes, workers and soldiers. In the termite

116 lineage,eusociality is thought to have evolved once, although the levels of social

117 complexity features, such as colony size, feeding habitat, symbiosis with

118 microorganisms and caste developmental pathways, diverged among termite

119 species. These characteristics are especially different between the two major

120 termite sublineages, i.e., the early-branching families (called "lower" termites) and

121 the most apical family Termitidae ("higher" termites) (Fig. 1a). To date, based on

122 the whole-genome sequences of a few termite species, the commonality and

123 diversity of genetic repertoires between Isoptera and Hymenoptera or between

124 termite lineages and their solitary outgroup (i.e., cockroach) have been investigated

$125(17,18)$. Additionally, in Zootermopsis nevadensis, clear differences in gene 
126 expression levels among castes (17) and in DNA methylation between alates and

127 workers (19) were detected.

Among the more than 2900 extant species of termites (Isoptera) (20), subterranean termites (Rhinotermitidae), especially two genera, Reticulitermes and Coptotermes, occupy an important evolutionary position (Fig 1a). Recent

132 phylogenetic studies showed that Rhinotermitidae is paraphyletic, and a clade

133 including Reticulitermes, Coptotermes and Heterotermes was shown to be sister to

134 Termitidae $(21,22)$. In particular, Reticulitermes exhibits intermediate social

135 complexity between those of higher (Termitidae) and lower (all the other families)

136 termites (23), for example, this genus displays primitive feeding ecology and gut

137 symbiont features, a relatively complex colony structure and a caste development

138 mode termed the bifurcated pathway (Fig. 1b). Moreover, Reticulitermes is the most

139 common termite group in palearctic (24) and nearctic (25) regions and a major pest

140 causing serious damage to human-made wooden structures (26). For these

141 reasons, members of this genus are probably among the most studied termites

142 (16). Nevertheless, despite their evolutionary, ecological and economic relevance,

143 subterranean termites remain an understudied group in terms of both genetics and

144 genomics.

In this study, we targeted the Japanese subterranean termite Reticulitermes

147 speratus. We conducted whole-genome sequencing, caste-specific RNA-seq

148 analysis and whole-genome bisulfite sequencing of $R$. speratus to understand the

149 genomic, transcriptomic and epigenetic bases of the social life of this termite

150 species. $R$. speratus nymphoids are almost exclusively produced

151 parthenogenetically by automixis with terminal fusion in primary queens, such that

152 the genome should be homozygous at most loci (27), which provides an advantage

153 in genome sequencing. We also compared the omics data of $R$. speratus with those

154 of sequenced higher and lower termites (Fig. 1a). Our integrative analyses of the

155 genome and transcriptome of $R$. speratus and other termites revealed that gene

156 duplications are often associated with caste-biased gene expression and caste-

157 specific functions, which highlights the significant role of gene duplication in

158 eusocial evolution in the termite lineage. 


\section{Results and Discussion}

\section{Genomic features of Reticulitermes speratus}

162 Genome sequencing of $R$. speratus was performed with genomic DNA isolated

163 from female secondary reproductives (nymphoids) [Fig. 1b]. R. speratus nymphoids

164 are almost exclusively produced parthenogenetically by automixis with terminal

165 fusion in primary queens, such that the genome should be homozygous at most loci

166 (27) and thus ease de Bruijn-graph-based genome assembly. We generated a total

167 of $86 \mathrm{~Gb}$ of Illumina HiSeq sequence data and assembled them de novo into 5817

168 scaffolds with an $\mathrm{N} 50$ of $1.97 \mathrm{Mb}$ and total size of $881 \mathrm{Mb}$ [Table 1], covering $88 \%$

169 of the genome based on the genome size $(1.0 \mathrm{~Gb})$ estimated by flow cytometry

170 (28). The assembled $R$. speratus genome has high coverage of coding regions,

171 capturing $99.2 \%$ (98.5\% complete; $0.7 \%$ fragmented) of 1367 Insecta

172 benchmarking universal single-copy orthologs (BUSCOs) (29) [Table 1]. The R.

173 speratus genome is rich in repetitive elements, which make up $40.4 \%$ of the

174 genome. A total of 15,591 protein-coding genes were predicted by combining the

175 reference-guided assembly of RNA-seq reads (36 libraries derived from different

176 castes, sexes and body parts; see below for details) and homology-based gene

177 prediction followed by manual curation of gene families of interest [Fig. 1c]. Whole-

178 genome bisulfite sequencing revealed extensive gene body methylation of the $R$.

179 speratus genome, amounting to $8.8 \%$ of methylated cytosines in the CG context

180 [Supplementary Fig. 1]. The genome-wide DNA methylation landscape was similar

181 to that of a dampwood termite $Z$. nevadensis (12\%) (19). These omics data and a

182 genome browser are available at http://www.termite.nibb.info/retsp/.

183 We compared the R. speratus gene repertoire with those of 88 other

184 arthropods, including the two termites $Z$. nevadensis and Macrotermes natalensis

$185(17,30)$. Ortholog analysis showed that $12,032(82.9 \%)$ of the 15,591 genes in $R$.

186 speratus genes were shared with other arthropods, and 1773 were taxonomically

187 restricted (TRGs) to Isoptera, among which 430 were shared with the other two

188 termites and 1343 were unique to $R$. speratus [Fig. 1c]. Whole-genome comparison

189 with two sequenced termites, M. natalensis and Z. nevadensis, showed a high

190 degree of synteny conservation [Fig. 1d]. We identified 2799 syntenic blocks (N50:

$191858.4 \mathrm{~kb}$ ) shared with M. natalensis that covered $95.1 \%$ of the $R$. speratus genome 
192 where $560.4 \mathrm{Mb}$ of nucleotides was aligned, while 3650 syntenic blocks (N50: 591.1

$193 \mathrm{~kb}$ ) shared with $Z$. nevadensis covered $72.1 \%$ of the $R$. speratus genome where

$194116.7 \mathrm{Mb}$ was aligned. Only a few cases of large genomic rearrangements were

195 found between termite genomes, at least, at the contiguity level of the current

196 assemblies, suggesting overall conservation of genome architecture in the lineage

197 of termites over 135 MY (Fig. 1a). Interestingly, despite such high conservation of

198 macrosynteny, interruptions or breaks in local synteny were observed and often

199 associated with tandem gene duplications. For example, when we examined

200 regions containing large tandem gene duplications (> 5-gene tandem duplications),

201 synteny between the $R$. speratus and $M$. natalensis genomes was interrupted in 10

202 of 21 regions (examples shown in Supplementary Fig 3).

\section{Transcriptome differentiation among castes}

205 Distinct castes arise from the same genome, a phenomenon called caste

206 polyphenism which is a distinctive hallmark in social insects $(31,32)$. To elucidate

207 caste-biased gene expression in order to understand the mechanism underlying the

208 caste-specific phenotypes, we compared the transcriptomes of three castes

209 (primary reproductives, workers and soldiers) in R. speratus [Fig. 1b;

210 Supplementary Table 2]. We sequenced 36 RNA-seq libraries, representing three

211 biological replicates of both sexes and two body parts ("head" and "thorax +

212 abdomen") for each of the three castes.

213 The results clearly showed that termite castes were distinctively

214 differentiated at the gene expression level. The multidimensional scaling (MDS) plot

215 depicted the three castes as clearly distinct transcriptomic clusters for both the

216 head and thorax + abdomen transcriptomes [Fig. 2a]. However, little sexual

217 difference was detected within each caste, although reproductives showed

218 substantial transcriptomic differences in thorax + abdomen samples between

219 queens and kings, probably due to the difference in the reproductive organs [Fig.

220 2a]. Using a generalized linear model (GLM) with caste and sex as explanatory

221 variables, we identified 1579 and 2076 genes differentially expressed among castes

222 in head and thorax + abdomen samples, respectively, with the criteria of a false

223 discovery rate (FDR)-corrected $P<0.01$, while we identified only 6 and 79 genes 
224 that were differentially expressed between sexes in the head and thorax +

225 abdomen samples, respectively, with the same criteria. We focused on the genes

226 that were differentially expressed among castes (caste-DEGs) and further classified

227 them into three categories of caste-biased genes (i.e., reproductive-, worker-, and

228 soldier-biased genes), with a criterion of $>2$-fold higher expression relative to that in

229 the other two castes. These caste-biased genes should account for the specialized

230 functions of each caste. Soldier samples exhibited the highest number of caste-

231 specific genes, suggesting the highly specialized functions of the soldier caste. This

232 is consistent with the finding of a previous RNA-seq analysis of the Eastern

233 subterranean termite Reticulitermes flavipes, reporting that a majority of DEGs were

234 soldier-specific (33); 73 of the 93 DEGs identified were up- or downregulated

235 specifically in the soldier caste. In addition to these soldier-specific $R$. flavipes

236 genes (e.g., troponin $C$ and fatty acyl-CoA reductase), the caste-biased genes

237 identified in our transcriptome analysis of $R$. speratus included genes previously

238 reported as caste-biased genes in other termites (34-37), e.g., vitellogenin

239 (reproductives), geranylgeranyl pyrophosphate synthase (soldiers), and beta-

240 glucosidase (probably associated with cellulase; workers). This consistency

241 between transcriptome analyses of different termite species indicates that the RNA-

242 seq analysis in this study is reliable and that the regulation and perhaps the

243 functions of these caste-biased genes are conserved across the termite lineage.

The caste-DEGs were enriched for Gene Ontology terms related to a wide

247 metabolism, hydrolase activity, oxidoreductase activity, lipid metabolism, signaling

248 and lysozyme activity. Protein motifs enriched in the caste-DEGs were also

249 identified, including cytochrome P450, lipocalin, lysozyme, glycosyl hydrolase

250 family, TGF-beta and trypsin [Supplementary Table 5]. Among the 1773

251 taxonomically restricted genes (TRGs) that were restricted to Isoptera (see above),

252 the termite-shared TRGs showed strong enrichment for caste-DEG (Fisher's exact

253 test, $\mathrm{P}<1.0 \mathrm{e}-7$ for head samples, $\mathrm{P}<1.0 \mathrm{e}-10$ for thorax+abdomen samples), while

254 the TRGs found only in $R$. speratus (orphan genes) did not $(P=0.99$ and $P=$

255 0.97, respectively). 
bioRxiv preprint doi: https://doi.org/10.1101/2021.07.11.451559; this version posted July 12, 2021. The copyright holder for this preprint (which

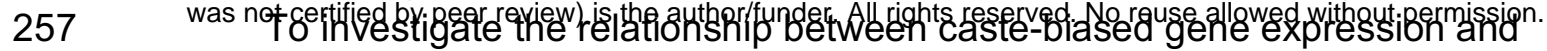

258 DNA methylation, we analyzed differential methylation levels among three castes

259 (reproductives, workers, and soldiers). The BS-seq data showed that the global

$260 \mathrm{CpG}$ methylation patterns were very similar among the castes [Supplementary Fig.

$2612 a b]$, in contrast to the methylation pattern of $Z$. nevadensis, in which DNA

262 methylation differed strongly between castes (winged adults vs. final-instar larvae)

263 and was strongly linked to caste-specific splicing (19). Instead, gene body DNA

264 methylation of $R$. speratus seems to be important for the expression of

265 housekeeping genes, as reported in the drywood termite Cryptotermes secundus

266 (18). Housekeeping genes exhibited a high degree of gene body methylation in all

267 castes of $R$. speratus, while caste-biased genes showed a significantly lower level

268 of DNA methylation [Supplementary Fig. 2cd].

\section{Gene duplication and caste-biased gene expression}

270 Evolutionary novelties are often brought about by gene duplications (38) (reviewed

271 in (39)), and the transition to eusociality in Hymenoptera has been associated with

272 gene family expansion $(18,40,41)$. Our ortholog analysis comparing the $R$.

273 speratus gene repertoire with those of 88 other arthropods identified 1396

274 multigene families duplicated in the R. speratus genome. Interestingly, compared to

275 the genome as a whole, the set of caste-DEGs identified above was significantly

276 enriched for genes in multigene families (X-squared $=218.62, \mathrm{df}=1, \mathrm{p}$-value $<$

277 2.2e-16). We also calculated the tau score as a proxy of caste specificity of gene

278 expression for all genes and found that duplicated genes were significantly more

279 caste-specific than single-copy genes ( $p<2.2 e-16$, Wilcoxon rank sum test) in both

280 transcriptome data sets (head and thorax+abdomen) [Fig. 2b]. Additionally, gene

281 set tests showed that sets of duplicated genes were differentially expressed in all

282 pairwise comparisons between castes [Fig. 2c]. These data highlight the important

283 roles of gene duplication in the caste evolution of termites. 
285 Multigene families related to caste-specific traits in $R$.

286 speratus

287 Caste-biased multigene families were associated with diverse functional categories,

288 some of which were strongly related to caste-specific behaviors and tasks. Here,

289 we highlight five families, namely, lipocalins (protein transporters for social

290 communication and physiological signaling), cellulases (carbohydrate-active

291 enzymes for worker wood digestion), lysozymes (immune-related genes for social

292 immunity), geranylgeranyl diphosphate synthases (metabolic enzymes for the

293 production of soldier defensive chemicals), and a novel termite-specific gene family

294 with unknown functions, as examples of multigene families relevant to termite

295 sociality. Molecular evolution studies have shown that the redundancy caused by

296 gene duplication may allow one paralog to acquire a new function

297 (neofunctionalization) or divide the ancestral function among paralogs

298 (subfunctionalization) $(38,39)$. We are particularly interested in the evolutionary

299 impact of gene duplication on caste specialization through neo/subfunctionalization.

300 Lipocalins

301 Lipocalins belong to a family of proteins, with molecular recognition properties such

302 as the ability to bind a range of small hydrophobic molecules (e.g. pheromones)

303 and specific cell surface receptors, and to form complexes with soluble

304 macromolecules (42). A previous study identified a gene of the lipocalin family,

305 SOL 1 , that is exclusively expressed in the mandibular glands of mature soldiers of

306 the rotten-wood termite Hodotermopsis sjostedti (43). SOL1 is thought to function

307 as a signaling molecule for defensive social interactions among termite colony

308 members (31). Moreover, RNA-seq analysis showed that a lipocalin gene, Neural

309 Lazarillo homolog 1 (ZnNlaz1), was specifically expressed in soldier-destined larvae

310 in an incipient colony of $Z$. nevadensis (44). Gene function and protein localization

311 analyses suggested that ZnNLaz1 was a crucial regulator of soldier differentiation

312 through the regulation of trophallactic interactions with a queen. Thus, it was of

313 interest that the lipocalin-related motif (Pfam PF00061; lipocalin/cytosolic fatty-acid

314 binding protein family) was significantly enriched in the list of caste-DEGs (FDR <

315 0.05; Supplementary Table 5). 
We identified 18 lipocalin family genes in the R. speratus genome [Fig. 3a-c;

317 Supplementary Table 7]. The number of lipocalin genes was larger than those in

318 other insects [Fig. 3c]. Phylogenetic analysis of lipocalin family genes identified in

319 arthropods, including three termite species, revealed a highly dynamic evolutionary

320 history of this protein family [Fig. 3a]. A couple of subfamilies, namely clades A and

$321 \quad B$, had experienced extensive expansion in the termite lineage. The most drastic

322 expansion was found in clade A, which includes $H$. sjostedti SOL1. In this clade, 7,

3239 and 5 genes were identified in R. speratus, M. natalensis and Z. nevadensis,

324 respectively, and extensive and independent gene expansions occurred in each

325 species. Clade B was also composed of genes with a termite lineage specific

326 expansion. In many cases, these lipocalin genes were found in tandem arrays in

327 the R. speratus genome [Fig. 3b]. The inferred phylogenetic tree indicated that

328 duplications in each clade occurred after the divergence of termites from a common 329 ancestor.

330 A comparison of the transcriptome among castes revealed that most

331 lipocalin genes (15 of 18) showed caste-biased gene expression [Fig. 2, Fig. 3ab].

332 The caste specificity, however, varied among genes, regardless of sequence

333 similarity and positional proximity on the genome. In particular, the expression

334 levels of genes in clades A and B drastically changed among castes. For example,

$335 R S 008823$ and RS008824 displayed solder-specific expression, the expression of

336 RS013912 was biased toward workers, and RS013913 was downregulated in

337 soldiers. RS008881 and RS008884 were exclusively expressed in queen bodies

338 (thorax + abdomen), while RS008882, a gene next to the aforementioned two

339 genes, showed quite different expression patterns and high expression levels in

340 heads, especially those of workers. These results indicate that termite lipocalin

341 genes underwent dynamic expansion in terms of gene repertoire, and regulatory

342 diversification of caste-biased expression. This gene expansion and regulatory

343 diversification of lipocalins may facilitate the evolution of the molecules involved in

344 signaling during caste development and among individuals through social

345 interactions.

346 To address the caste-specific function of the lipocalin paralogs, the

347 expression patterns of several selected caste-biased lipocalin genes were

348 examined by in situ hybridization [Fig. 3d-h, Supplementary Fig. 6]. RS008881, a

349 queen-biased lipocalin gene, was found to be expressed exclusively in the

350 accessory glands of the ovary [Fig. 3d]. The next gene on the same scaffold, 
351 RS008882, was shown to be specifically expressed in worker antennae and

352 maxillary/labial palps [Fig. 3f-h]. RS008823, a soldier-biased gene, was expressed

353 exclusively in the frontal gland cells of the soldier heads [Fig. 3e]. Note that ovaries

354 and frontal glands develop during postembryogenesis in a caste-specific manner

355 (i.e., ovaries in queens and frontal glands in soldiers) in the pathway of caste

356 differentiation in $R$. speratus. Antennae and maxillary/labial palps are not caste-

357 specific but crucial sensory organs, especially for blind termite immatures, such as

358 workers. Given that animal lipocalins generally work as carrier proteins (45), there

359 is a possibility that focal termite lipocalins bind and convey some molecules to the

360 targets from caste-specific organs (e.g., egg-recognition pheromone and soldier

361 defensive and/or inhibiting substances; (46-48)), or participate in sensory

362 reception, such as the role of odorant-binding proteins (49).

\section{Cellulases}

364 Lignocellulose degradation in termites is achieved by a diverse array of

365 carbohydrate-active enzymes (CAZymes) produced by the host and their intestinal

366 symbionts. The repertoire of CAZyme families in the genome of $R$. speratus did not

367 show considerable differences from those of other nonxylophagous insects, such as

368 a honeybee and a fruit fly (Supplementary Fig. 4). However, we found gene family

369 expansion and expressional diversification for glycoside hydrolase family $(\mathrm{GH}) 1$

370 and $\mathrm{GH} 9$ members. The majority of $\mathrm{GH} 1$ and $\mathrm{GH} 9$ members are $\beta$-glucosidase

371 (BGs; EC 3.2.1.21) and endo- $\beta$-1,4-glucanases (EGs; EC 3.2.1.4), respectively,

372 which are essential for cellulose digestion in termites (50).

373 We identified $16 \mathrm{GH} 1$ paralogs [Supplementary Table 8]. Such gene expansion

374 of $\mathrm{GH} 1$ was also observed in the genome of other termites, but the reason for the

375 gene expansion remains elusive (51). Although the phylogenetic tree divided these

$376 \mathrm{GH} 1$ paralogs into four distinct groups (clades A to D in Fig. 4a), most of them were

377 tandemly located in the genome of $R$. speratus (Fig. $4 \mathrm{bc}$ ). The predominantly

378 expressed BG gene was RS004136, while the expression of this gene was clearly

379 biased toward the bodies (thorax + abdomen) of reproductives and workers (Fig.

380 4b). This gene formed a rigid clade with bona fide BGs reported from the salivary

381 glands or midgut of termites (clade A in Fig. 4a) (52), suggesting that this gene is

382 involved in cellulose digestion in $R$. speratus. Indeed, in situ hybridization analysis

383 showed that RS004136 was specifically expressed in the salivary glands of workers 
384 (Fig. 4de, Supplementary Fig. 7a). Other GH1 members showed a wide variety of 385 expression patterns across castes and body parts [Fig. 4b]. Some of them might 386 have diversified their functions, other than wood digestion, related to termite 387 sociality, such as egg-recognition pheromones (53). A typical example displaying 388 such diversification was RS004624, which was expressed specifically in the 389 abdomens of queens [Fig. 4b]. The peptide sequence of this gene showed a 390 monophyletic relationship with that of Neofem2 of Cryptotermes secundus (clade D 391 in Fig. 4a), which is a queen recognition pheromone probably functioning in the 392 suppression of reproductive emergence (54). In situ hybridization showed that $393 R S 004624$ was specifically expressed in the accessory glands of queen ovaries 394 (Fig. 4fg, Supplementary Fig. 7b), suggesting that RS004624 is involved in 395 enzymatic activities in queen-specific glands. Together with the results for a queen396 biased lipocalin (RS008884), this finding indicates that the queen accessory glands 397 may produce some queen-specific pheromones. Like lipocalins, GH1 paralogs are 398 also typical examples of multigene family members participating in caste-specific 399 tasks, which may be acquired by gene duplication resulting in neo- or 400 subfunctionalization.

401 We found four paralogs of GH9 in R. speratus [Supplementary Fig. 5,

402 Supplementary Table 8]. Although several insect GH9 EGs have acquired the 403 ability to hydrolyze hemicellulose (55), neo- or subfunctionalization of termite EGs 404 has yet to be clarified. Intriguingly, we found that the GH9 member RS006396 was 405 weakly but uniformly expressed across all termite body parts and castes. This result 406 suggests that some GH9 members also perform a function other than that of 407 cellulase, as is the case for $\mathrm{GH} 1$.

\section{Lysozymes}

409 The immune system of termites is of particular interest, because the group living of 410 termites with nonsclerotized and nonpigmented epidermis and microbe-rich habitat 411 puts them at high risk for pathogenic infections (56). Thus, defense against

412 pathogenic microbes is important for termites. In the R. speratus genome we 413 identified 251 immune-related genes [Supplementary Information 1.9,

414 Supplementary Table 26]. The repertoire and number of immune-related genes of $415 R$. speratus showed no large differences compared to those of other insect species, 416 but a notable exception was found for lysozymes [Supplementary Fig. 8]. 
417 Lysozymes are involved in bacteriolysis through hydrolysis of $\beta-1,4$-linkages in the

418 peptidoglycans present in bacterial cell walls, and three distinct types of lysozymes,

419 chicken- or conventional-type (c-type), goose-type (g-type), and invertebrate-type (i-

420 type) lysozymes, have been found in animals (57). We identified 13 and 3 genes

421 encoding c-type and i-type lysozymes, respectively, and the number of lysozyme

422 genes was larger than those in other insects [Fig. 5, Supplementary Table 9].

423 Phylogenetic analysis revealed that c-type lysozymes underwent extensive gene

424 duplications in the sublineage leading to $R$. speratus [Fig. 5a]. Seven c-type

425 lysozymes formed a tandem array on scaffold_859 [Fig. 5b], probably generated by

426 repeated tandem gene duplication events. Interestingly, most of the c-type

427 lysozyme genes showed caste-biased expression. Three genes (RS014698,

$428 R S 100022$, and RS100023) exhibited high expression levels compared to those of

429 other lysozyme genes and were expressed in a soldier-specific manner, while

$430 \quad R S 100026$ was expressed in a worker-specific manner and RS100024 and

431 RS100025 were highly expressed in both workers and soldiers [Fig. 5b]. The

432 differential expression patterns of the lysozyme genes in $R$. speratus may represent

433 division of labor among castes in terms of colony-level immunity.

434 It is also possible that duplicated lysozymes may have functions other than

435 immunity. A previous study indicated that the salivary glands of $R$. speratus secrete

436 c-type lysozymes to digest bacteria ingested by termites through social feeding

437 behavior (58). The same lysozyme genes are also expressed in the queen ovaries

438 and eggs and play a role in egg recognition as proteinaceous pheromones in $R$.

439 speratus $(48,53)$. We could not find identical sequences of these lysozyme genes

440 in our gene models, but these sequences were most closely related to RS002400

441 with $88 \%$ nucleotide identity, which occupied the basal position of the lineage-

442 specific gene expansion (Fig. 5a).

\section{GGPP synthase}

444 Whole-genome comparison of $R$. speratus with $Z$. nevadensis and $M$. natalensis

445 revealed a $270 \mathrm{~kb} R$. speratus-specific fragment in scaffold_31, while the rest of this

446 scaffold showed very high syntenic conservation among the three termites [Fig 6a].

447 We found that the R. speratus-specific region was encompassed by a tandemly

448 duplicated gene cluster composed of 13 genes encoding geranylgeranyl

449 diphosphate (GGPP) synthase [Fig. 6b, Supplementary Table 10]. GGPP synthase 
450 catalyzes the consecutive condensation of an allylic diphosphate with three

451 molecules of isopentenyl diphosphate to produce GGPP, an essential precursor for

452 the biosynthesis of diterpenes, carotenoids and retinoids (59-61). The extensive

453 duplication of GGPP synthase paralogs observed in $R$. speratus is unusual

454 because the genomes of other insects surveyed have only a single copy of GGPS

455 synthase gene. The phylogenetic analysis of GGPP synthase homologs revealed

456 two clusters, a possibly ancestral group (including RS007484) and an apical group

457 (including other paralogs identified) [Fig. 6c]. The latter cluster also contained some

458 GGPP synthase paralogs obtained from the termitid Nasutitermes takasagoensis

$459 \quad(34)$

460 Transcriptome data indicated that all of the GGPP synthase genes, except

461 RS007484 which was a member of the ancestral group in the phylogenetic tree,

462 showed caste-biased expression, and caste specificity varied across the paralogs

463 [Fig 6b]. Specifically, RS100010, RS007480, RS100012, RS100015, RS100016,

464 RS100017 and RS007483 showed soldier-specific expression, while RS007481,

$465 R S 007482$ and RS100013 showed reproductive-specific expression [Fig 6b].

466 Several GGPP synthase genes have been identified in some termite species and

467 are known to function in a caste-specific manner; for example, the soldiers of $N$.

468 takasagoensis synthesize defensive polycyclic diterpenes by high expression of the

469 GGPP synthase gene in the frontal gland to use chemical defense (62). It has been

470 reported that the soldiers of Reticulitermes have a frontal gland in which diterpenes

471 are synthesized, although the biological role is not fully understood (63-65).

472 Consequently, it is possible that the soldier-specific GGPP synthases identified to

473 date are involved in chemical defense. Indeed, in situ hybridization revealed that

474 the soldier-specific GGPP synthase RS100016 was expressed exclusively in the

475 soldier frontal gland, as shown in a previous study (66) [Fig. 6d, Supplementary Fig.

476 7c]. It is also possible that reproductive-specific GGPP synthases are involved in

477 the metabolism of other diterpenes, such as pheromone synthesis, especially

478 RS007481, which shows strong queen-specific expression in the thorax and

479 abdomen and may play a role in the synthesis of queen substances.

$480 \quad$ Under the branch-site (BS) model of codon substitutions (67), significant

481 positive selection was detected on five branches of $R$. speratus GGPP synthase

482 family tree [Fig. 6e]: ancestral branches \#1 and \#2, and the branches leading to

483 RS100017 (branch \#3), RS100012 (branch \#4) and RS007483 (branch \#5). These

484 results suggest that all GGPP synthase paralogs of $R$. speratus except the 
ancestral type RS007484 have experienced positive selection and finally acquired novel roles for the production of defensive and/or pheromonal substances.

\section{The TY family, a novel gene family restricted to termites}

489 Numerous studies have shown that novel genes (e.g., TRG) play important roles in

490 the evolution of novel social phenotypes in hymenopteran social insects $(8,68,69)$.

491 We found that termite-shared TRGs showed strong enrichment for caste-DEGs

492 (see above). A striking example of caste-biased TRGs is a tandem array of three

493 novel genes [Fig. 7a; Supplementary Table 11], RS001196, RS001197 and

494 RS001198, that have no significant homologs in any organisms outside termite

495 clades. These three genes were expressed at extremely high levels (up to 250,000

496 RPKM), which constituted approximately $30 \%$ of the worker head transcriptome,

497 and strongly biased across the three castes [Fig. 7a]. Each gene was composed of

498 a single exon encoding a short peptide $\sim 60$ aa in length that contained a secretion

499 signal peptide in the N-terminal region followed by a middle part rich in charged

500 amino acid residues and C-terminal part rich in polar amino acids with unusually

501 high number of tyrosine residues [Fig. 7b]. Here, we named this novel class of

502 peptides the termite-specific tyrosine-rich peptide family (TY family). The three TY

503 genes showed modest sequence similarity with each other, suggesting that they are

504 paralogs derived by tandem duplication. TY family orthologs were also found in the

505 genomes of $Z$. nevadensis and M. natalensis [Fig. 7b]. We estimated pairwise

506 evolutionary rates (the ratio of nonsynonymous to synonymous substitutions, i.e.,

$507 \mathrm{dN} / \mathrm{dS}$ ) between $R$. speratus and $Z$. nevadensis for these three peptides. The

$508 \mathrm{dN} / \mathrm{dS}$ for each gene ranged from 0.03 to 0.16 (Fig. 7c), indicating that they evolved

509 under strong purifying selection and suggesting a conserved function in the termite

510 lineage. Indeed, Z. nevadensis orthologs were also expressed at a high level in the

511 soldier and worker castes in a pattern similar to that in $R$. speratus.

\section{Facilitation of caste specification by gene duplication}

513 Recent advances in sociogenomics in different social insects are promoting our

514 understanding of the genetic bases of social evolution, which include the co-option 
515 of genetic toolkits of conserved genes, changes in protein-coding genes, cis-

516 regulatory evolution leading to genetic network reconstruction, epigenetic

517 modifications and TRGs (15, 70). In addition to these components, our genomic

518 and transcriptomic analyses in $R$. speratus highlighted the significance of gene

519 duplication for caste specialization. Gene duplication is, in general, a key source of

520 genetic innovation that plays a role in the evolution of phenotypic complexity; gene

521 duplication allows for subsequent divergent evolution of the resultant gene copies,

522 enabling evolutionary innovations in protein functions and/or expression patterns

523 (71-73). Regarding eusocial evolution in insects, Gadagkar (74) first pointed out the

524 importance of gene duplication; 'genetic release followed by diversifying evolution'

525 made possible the appearance of multiple caste phenotypes in social insects. Many

526 decades later, genomic analyses revealed gene family expansion, especially in

527 relation to chemical communication, in both ants (odorant receptors; (75-77)) and

528 termites (ionotropic receptors; (18)). Based on Godagkar's hypothesis, duplicated

529 genes can be released from the constraints of original selection, leading to new

530 directional evolution, i.e., for caste-specific functions (e.g., queen- or worker-trait

531 genes). However, the detailed roles and significance of gene duplication in social

532 evolution have been elusive.

533 This study revealed that gene duplication associated with caste-biased gene

534 expression is prevalent in the $R$. speratus genome. The list of duplicated genes

535 encompasses a wide array of functional categories related to the social behaviors in

536 termites as exemplified by transporters such as lipocalins (communication and

537 physiological signaling; cf. $(31,44))$, digestive enzymes such as carbohydrate-

538 active enzymes, immune-related genes such as lysozymes (social immunity), and

539 metabolic enzymes such as GGPP synthase (social defense). This study

540 demonstrated that caste-specific expression patterns differed among in-paralogs.

541 Although such paralogous genes were often observed in tandem in the genome,

542 the expression patterns were often independent from one another, showing

543 differential caste biases in many cases. Additionally, discordant caste biases in

544 transcriptional expression were observed among closely related paralogs with

545 similar coding sequences, as represented by little correlation between phylogenetic

546 position and caste specificity (Fig. 3a). Although the regulatory and evolutionary

547 mechanisms underlying caste-biased expression patterns are elusive, these

548 examples strongly suggest that gene duplications have facilitated caste

549 specialization, leading to social evolution in termites. 
After the gain of caste-biased gene regulation, subfunctionalization and/or neofunctionalization seems to have occurred, leading to caste-specific expression

552 and caste-specialized functions. For example, in the case of lipocalin family,

553 lipocalin paralogs were generated by lineage-specific functional expansion in caste-

554 specific organs or tissues: a queen-specific lipocalin (RS008881) was expressed

555 specifically in the ovarian accessory glands, while a soldier-biased lipocalin

556 (RS008823), was expressed exclusively in the frontal glands in soldier heads [Fig.

557 3de]. Taken together, we hypothesize that, in termites, caste specification through

558 gene duplication proceeds by the following three steps: 1) gene family expansion by

559 tandem gene duplication, 2) regulatory diversification leading to an expression

560 pattern restricted to a certain caste, and 3) subfunctionalization and/or

561 neofunctionalization of the gene products conferring caste-specific functions. As an

562 exaptation of these steps, the case in which one (or some) of the multiple functions

563 of pleiotropic genes are allocated and specialized to a duplicated gene copy might

564 have led to caste-specific subfunctionalization $(38,39)$.

565 Recently, it was suggested that the evolution of phenotypic differences

566 among castes in the honey bee was associated with the gene duplication, by

567 showing that duplicated genes had higher levels of caste-biased expression

568 compared to singleton genes (78). It was also shown that the level of gene

569 duplication was correlated with social complexity in bees (superfamily Apoidea)

570 (78). Given the independent origin of eusociality in termites and honeybees, gene

571 duplications might be a shared mechanism facilitating the evolution of caste

572 systems in social insects.

573

574

\section{Materials and Methods}

$576 \quad$ Insects

577 All mature colonies of Reticulitermes speratus used for genome, RNA, and Bisulfite

578 sequencing (BS-seq), were collected in Furudo, Toyama Prefecture, Japan

579 [Supplementary Table 1]. Detailed sample information is described in SI Appendix,

580 Supplementary Methodology. 
581 Sample collection, genome sequencing and assembly

582 All colonies of Reticulitermes speratus used for genome, RNA, and bisulfite

583 sequencing were collected at Furudo, Toyama Prefecture, Japan.Detailed sample

584 information is described in Supplementary Table 1 and SI Appendix,

585 Supplementary Methodology.

586

We used female secondary reproductives (nymphoids I and II) for genome sequencing. We excluded the gut and ovaries of nymphoids to avoid contamination

589 by DNAs from the king or other microorganisms. Genomic DNA was isolated from 590 each individual using a Genomic-tip 20/G (Qiagen). We used 5 microsatellite loci

591 (Rf6-1, Rf21-1, Rf24-2, Rs02, and Rs03) to confirm whether they were homozygous

592 at these loci and shared the same genotype. The purified genomic DNA purified

593 was fragmented with a Covaris S2 sonicator (Covaris), size-selected with

594 BluePippin (Sage Science), and then used to create two pair-end libraries using a

595 TruSeq DNA Sample Preparation Kit (Illumina) with insert sizes of $\sim 250$ and $\sim 800$

596 bp [Supplementary Table 3]. Four Mate-pair libraries with peaks at $\sim 3 \mathrm{~kb}, \sim 5 \mathrm{~kb}, \sim 8$

$597 \mathrm{~kb}$ and $\sim 10 \mathrm{~kb}$, respectively, were also created using a Nextera Mate Pair Sample

598 Preparation Kit (Illumina) [Supplementary Table 3].These libraries were sequenced

599 using an Illumina HiSeq system with $2 \times 151$ bp paired-end sequencing protocol.

600 Reads of the pair-end and mate-pair libraries were assembled using ALLPATHS-

601 LG (build\# 47878), with default parameters. BUSCO

602 v4.0.6(29)(https://busco.ezlab.org/) was used in quantitative measuring for the

603 assessment of genome assembly using insecta_odb10 as the lineage input. A

604 genome browser was built using JBrowse (https://jbrowse.org/)

\section{Gene prediction}

606 A protein-coding gene reference set was generated with two main sources of

607 evidence, aligned $R$. speratus transcripts and aligned homologous proteins of other

608 insects, and a set of ab initio gene predictions. RNA-seq reads were assembled de

609 novo using Trinity, and then mapped to the genome using Exonerate. We

610 processed homology evidence at the protein level using the reference proteomes of

6117 sequenced insects including $Z$. nevadensis and Blattodea protein sequences

612 predicted from RNA-seq of Periplaneta americana and Nasutitermes

613 takasagoensis. These proteins were split-mapped to the R. speratus genome with 
614 Exonerate. These models were merged using the EvidenceModeler (EVM), which

615 yielded 15584 gene models. Seventy-four genes were manually inspected and

616 corrected. In particular, tandemly duplicated genes were liable to be incorrect gene

617 prediction with erroneous exon-exon connections across homologs. The final set of

61815591 genes was designated as Rspe OGS1.0 [Supplementary Data 2

619 (DOI:10.6084/m9.figshare.14267381)]. The quality of theOGS1.0 was evaluated by

620 assessing two types of evidence, homology and expression. Among 15591 genes,

$62112996(83.3 \%)$ showed any hits in the NCBI nr database, $10440(70.0 \%)$ included

622 known protein motifs defined in the Pfam database, and 14302 (91.7\%) showed

623 evidence of expression with a threshold of RPKM $=1.0$ in any sample of caste-

624 specific RNA-seq data. In sum, 15577 (99.9\%) had evidence for the presence of

625 homologs and/or expression.

\section{Orthology inference and gene duplication analysis}

627 Orthology determination among three termites: Orthologous genes among the

628 proteomes of three termite species, R. speratus, Z. nevadensis, and M. natalensis

629 (gene models RspeOGS1.0, ZnevOGSv2.229, and MnatOGS3, respectively), were

630 determined by pairwise comparisons with InParanoid v4.1 followed by three-

631 species comparison with MultiParanoid. M. natalensis gene set, MnatOGS3, was

632 built in this study using a similar pipeline as used for $R$. speratus.

633 Ortholog analysis with arthropod proteomes: Orthology relationships of $R$. speratus

634 genes (OGS1.0) with other arthropod genes were analyzed by referring to the

635 OrthoDB gene orthology database ver.8 (87 arthropod species)

636 (https://www.orthodb.org/). We grouped R. speratus genes with the OrthoDB

637 ortholog group using a two-step clustering procedure. For each $R$. speratus protein,

638 BLASTP was used to find similar proteins among the arthropod proteins, and the

639 ortholog group of the top hit was provisionally assigned to the query $R$. speratus

640 gene. Then, the ortholog grouping was evaluated by comparing the similarity level

641 (BLAST bit score) among members within the focal ortholog group. We keep the

642 grouping if the BLAST bit score between the query $R$. speratus gene and top

643 arthropod gene was higher than the minimal score within the original cluster

644 members. Among 15591 R. speratus OGS1.0 genes, 12434 were clustered into

6459033 OrthoDB Arthropod ortholog groups. Gene duplication was assessed based

646 on this clustering. If two or more members of one species were included in a single

647 ortholog group, they were regarded as a multigene family. 


\section{RNA-seq}

649 W4-5 workers (old workers) and soldiers were collected from each colony. To 650 collect primary reproductives, dealated adults were chosen randomly from each 651 colony in accordance with the method of the previous literature (79), and female-

652 male pairs were mated (Supplementary Table 1). Kings and queens were sampled

653 after 4 months. Each individual was divided into head and body parts (thorax +

654 abdomen). We prepared RNA-seq libraries for 12 categories based on castes

655 (reproductives, workers and soldiers), sexes (males and females) and body parts

656 (head, and thorax + abdomen). Three biological replications of the 12 categories

657 were made with three different field colonies totaling 36 RNA-seq libraries

658 [Supplementary Table 2]. All Illumina libraries prepared using a TruSeq Stranded

659 mRNA Library Prep kit were subjected to a single-end sequencing of $101 \mathrm{bp}$

660 fragments on HiSeq 2500. The cleaned reads were mapped onto the genome with

661 TopHat v2.1.0 guided by the OGS1.0 gene models. Transcript abundances were

662 estimated using featureCounts and normalized with the trimmed mean of M-values

663 (TMM) algorism in edgeR. Differentially expressed genes among castes and

664 between sexes were detected in each body part (head / thorax and abdomen) using

665 a generalized linear model with two factors, namely, caste and sex using edgeR

666 with the conditions set as false discovery rate (FDR) $<0.01$ and the log2 fold

667 change of the expression level $>1$.

668 Data Availability

669 Data from whole-genome sequencing, transcriptome sequencing, and methylome

670 sequencing have been deposited in the DDBJ database under BioProject

671 accessions PRJDB2984, PRJDB5589 and PRJDB11323, respectively. The

672 analyzed data including genome assembly, gene prediction, annotation, and gene

673 expression are available through Figshare

674 (https://doi.org/10.6084/m9.figshare.c.5483235). The R. speratus genome browser

675 is available at http://www.termite.nibb.info/retsp/.

676 Code availability

677 Custom R and Ruby scripts were deposited into Github

678 (https://github.com/termiteg/retsp_genome_paper). 


\section{Acknowledgments}

681 We thank R. H. Suzuki and A. Karasawa for experimental support, T. Nishiyama

682 and M. Hasebe for discussion on genome analyses, N. Kanasaki and K. Kai for

683 rearing insects, T. Shibata, S. Ohi, T. Aizu, H. Ishizaki, H. Asao for next-generation

684 sequencing (NGS), and K. Yamaguchi for NGS data management. Computations

685 were partially performed on the supercomputers at the Data Integration and

686 Analysis Facility, National Institute for Basic Biology. This study was funded by the

687 JSPS/MEXT KAKENHI Grant Numbers 25128705, 24570022, 16K07511,

688 JP19H03273, 22128008, 19K22294, 22150002 and NIBB Collaborative Research

689 Programs (20-323).

690

691

692 Author contributions

693 S.S., Y.H., T.M., and K.M. designed and managed the project. D.W., K.T., R.S.,

694 H.Y., Y.M., R.S., and K.M. collected samples. D.W., R.S., Y.M., and R.S. performed

695 the DNA extraction. S.S., and A.T. performed the library construction and genome

696 sequencing. Y.H., D.W., K.T., R.S., H.Y., and Y.M. generated the RNA-Seq data.

697 S.S., Y.H., and R.S. generated the BS-Seq data. S.S., Y.H., D.W., G.T., M.Y.H,

698 K.T., M.M., Y.S., K.O., T.N., H.G., M.K.H., and S.M. contributed to the genome

699 assembly and annotation. S.Su, and M.K. performed histological analyses. S.S.,

700 Y.H., G.T., T.M., and K.M. drafted the manuscript. All authors contributed to the

701 final version of the manuscript. 


\section{Tables}

704 Table 1. Summary of Reticulitermes speratus genome assembly, annotation 705 and methylome

\begin{tabular}{|l|l|c|}
\hline Genome & No. scaffolds & 5,817 \\
\hline & No. contigs & 63,310 \\
\hline & Total length & $881 \mathrm{Mb}$ \\
\hline & Scaffold N50 & $1,967 \mathrm{~kb}$ \\
\hline & Contig N50 & $37.5 \mathrm{~kb}$ \\
\hline & Longest scaffold & $14.3 \mathrm{Mb}$ \\
\hline & GC\% & $39.70 \%$ \\
\hline & No. Ns & $63 \mathrm{Mb}$ \\
\hline & Completeness (BUSCO & C:98.5\% [S:98.1\%, D:0.4\%] \\
\hline & insecta_odb10) & 15,591 \\
\hline Annotation & No. genes (coding) & $40.4 \%$ \\
\hline & Repeat content & $8.79 \%$ \\
\hline & & \\
\hline Methylome & \%methylated CpG & \\
\hline & & \\
\hline
\end{tabular}




\section{Figure legends}

708 Figure 1: Phylogenetic position of Reticulitermes speratus in Blattodea, its

709 developmental pathway, and evolution of the gene repertoire and genome

710 structure.

711 (a) Phylogenetic tree of termites and cockroaches. Estimated divergence dates

712 (mya: million years ago) are based on Bucek et al. (80). R. speratus is marked in

713 red, and two termites mainly compared in this study are marked with bold

714 characters. (b) Developmental pathway of $R$. speratus. There are 2 larval stages

715 before the molt into a nymph (with wing buds) or worker (no wing buds). There are

7166 imaginal stages, and the 6th-stage nymphs molt into alates, which are primary

717 reproductives (queen and king). Secondary reproductives (neotenics) differentiate

718 from the 3rd- to 6th-stage nymphs. In the apterous line, there are at least 5 stages

719 of workers. Some workers in the colony molt into presoldiers and soldiers. Female

720 neotenics used for genome sequencing and 3 castes used for RNA-seq are marked

721 with asterisks. (c) Gene repertoire of $R$. speratus categorized by orthology. $R$.

722 speratus genes were compared to those of 88 arthropods and grouped into three

723 classes: orthologs shared with other arthropods (labeled 'arthropod'), orthologs

724 shared with other termites (Z. nevadensis and/or M. natalensis) but with no

725 orthologs in other arthropods (labeled 'termite'), and orphan genes unique to $R$.

726 speratus (labeled 'no hit'). (d) High conservation of synteny between termite

727 genomes revealed by dot plots generated by comparing $R$. speratus with $Z$.

728 nevadensis and $M$. natalensis. Scaffolds longer than $2.0 \mathrm{Mb}$ in the $R$. speratus

729 assembly are used for plotting. Forward alignments are plotted in red and reverse

730 alignments are plotted in blue.

731

732 Figure 2: Caste-specific transcriptome analysis and the enrichment of 733 duplicate genes for caste-biased genes.

734 (a) Multidimensional scaling (MDS) plot of RNA-seq data showing relatedness

735 between the expression profiles of different castes (reproductive, soldier and

736 worker) and sexes (male and female). The left panel plots RNA-seq data from head

737 samples, and the right panel plots data from thorax + abdomen samples. Three

738 biological replicates were analyzed for each condition and plotted individually. (b)

739 Numbers of caste-biased genes with >2-fold higher expression levels than the other 
740 two castes. Colours in each bar indicate the differences of RNA-seq data obtained.

741 (c) Violin plots showing the distribution of tau indexes of duplicate genes and single

742 genes. Tau values range between 0 and 1, with low values indicating invariable and

743 constitutive expression between castes and higher values supporting caste

744 specificity. In both body part samples, the tau values of duplicate genes were

745 significantly greater than those of single genes $(p<2.2 e-16$, Wilcoxon rank sum

746 test). (d) Enrichment of caste-DEGs (differentially expressed genes among castes)

747 for duplicate genes. In each comparison between castes (soldier vs worker,

748 reproductive vs worker, and reproductive vs soldier), all genes are ranked and

749 ordered by log-fold-change value along the horizontal axis. Black bars mark the

750 positions of genes. Genes of sociality-related functions highlighted in the text are

751 selected and plotted in the lower panels. Curved lines in the upper panel show

752 relative enrichment of the duplicate genes (blue line) or single genes (green line)

753 relative to uniform ordering.

755 Figure 3: Lipocalin genes in R. speratus.

756 (a) Maximum likelihood (ML) tree of lipocalin homologs based on the amino acid 757 sequences obtained with a log gamma (LG) model. Branches leading to clade $A$ 758 and clade $B$, which show gene family expansion specific to termite sublineages, are 759 marked in yellow and green, respectively. Color gradients in the outer tracks show 760 the expression levels as averaged log(RPKM+1) values in three castes

761 (reproductive, soldier, and worker). Expression levels of head samples and thorax + 762 abdomen samples are shown in purple and green, respectively. Caste-DEGs

763 (differentially expressed genes among castes) are marked as R, S, or W beside the 764 color gradients, indicating biases toward the reproductive, soldier, or worker caste, 765 respectively. (b) Lipocalin multigene clusters in the $R$. speratus genome and their 766 relative expression levels among castes. The heatmap shows the Z-scores of the $767 \log (\mathrm{RPKM}+1)$ values in the caste-specific transcriptome. (c) Comparison of the 768 number of lipocalin subclasses among representative arthropods. Note clades $\mathrm{A}$ 769 and $B$ are specific to termites. (d) Vertical cryosection of the queen abdomen 770 subjected to in situ hybridization with an antisense DIG-labeled RS008881 mRNA 771 probe. The accessory gland cell layer is stained dark (arrowhead), in contrast to the

772 other ovarian tissues, including the spermatica (asterisk). Bar $=0.2 \mathrm{~mm}$. (e)

773 Photographs of in situ hybridization for RS008823 mRNA in the soldier head. The

774 front of the head is on the left side. The gland cell layer surrounding the frontal 
775 gland reservoir $(R)$ is stained dark (arrowhead). The asterisk indicates the brain.

776 Bar $=0.1 \mathrm{~mm}$. (f, $\mathbf{g}, \mathbf{h})$ Vertical cryosection of the worker antenna (f) and horizontal

777 cryosections of the worker labial palp (g, right palp) and maxillary palp (h, the last

778 segment of the left (upper) and right (lower) palp) subjected to in situ hybridization

779 for RS008882 mRNA. Tissues around some sensilla are stained dark (arrowhead).

780 Bar $=0.1 \mathrm{~mm}$. Photographs of cryosections hybridized with sense probes (negative

781 controls) are shown in Supplementary Fig. 6a-c.

783 Figure 4: Glycoside hydrolase family (GH) 1 in the $R$. speratus genome.

784 (a) ML tree of $\mathrm{GH} 1$ genes based on the amino acid sequences obtained with a

$785 \mathrm{LG}+\mathrm{G}+\mathrm{I}$ model. Fourteen of $16 \mathrm{GH} 1$ genes in $R$. speratus were used; two genes

786 (RS004146 and RS100005) were removed from the analysis due to incomplete

787 retrieval of the coding sequences from gapped scaffolds. GH1 subclasses are

788 colored and labeled A, B, C, and D. (b) GH1 multigene clusters in the R. speratus

789 genome and their expression levels. Letters A-D on the gene structures represent

$790 \mathrm{GH} 1$ subclasses categorized in the phylogenetic tree in (a). The heatmap shows

791 the Z-scores of the $\log (\mathrm{RPKM}+1)$ values in the caste-specific transcriptome. (c)

792 Synteny comparison around the $\mathrm{GH} 1$ multigene cluster region (orange rectangle)

793 between R. speratus and M. natalensis genomes. (d) Vertical cryosection of the

794 worker thorax subjected to in situ hybridization with an antisense DIG-labeled

795 RS004136 mRNA probe. The head part is on the right side. Bar $=0.2 \mathrm{~mm}$. (e)

796 Magnified view of the worker thorax. The salivary gland cells are specifically stained

797 dark (arrowhead). Bar $=0.1 \mathrm{~mm}$. (f) Vertical cryosection of the queen abdomen

798 subjected to in situ hybridization for RS004624 mRNA. Bar $=0.2 \mathrm{~mm}$. (g) Magnified

799 view of the queen ovary. The accessory gland cell layer is stained dark

800 (arrowhead), in contrast to the other ovarian tissues, including ovarioles with two

801 oocytes (asterisks). Bar $=0.1 \mathrm{~mm}$. See Supplementary Fig. 7a-b for negative

802 controls of the in situ hybridization experiments (d-g).

804 Figure 5: Lysozyme family in the R. speratus genome.

805 (a) ML tree of lysozyme genes with a GTR+G model. The red curve indicates a

806 lineage-specific gene expansion observed in the $R$. speratus genome for a c-type

807 lysozyme. (b) Lysozyme multigene clusters in the R. speratus genome and their

808 relative expression levels among castes. The heatmap shows the Z-scores of the

$809 \log (\mathrm{RPKM}+1)$ values in the caste-specific transcriptome. 
811 Figure 6: Geranylgeranyl diphosphate (GGPP) synthase homologs in the $\boldsymbol{R}$. 812 speratus genome.

813 (a) Synteny comparison around GGPP synthase loci among three termites, $R$.

814 speratus, M. natalensis and $Z$. nevadensis. R. speratus-specific insertions were

815 found, where GGPP synthase paralogs were tandemly duplicated in the $R$. speratus

816 genome. (b) Genomic location and gene expression of $R$. speratus GGPPS

817 homologs. The heatmap shows the expression level calculated by mean-centered

$818 \log (\mathrm{RPKM}+1)$. Yellow indicates high expression, while blue denotes low expression.

819 Black represents the mean level of expression among the castes. Note that the

820 heatmap of RS007484 is almost entirely black for all samples, which indicates that

821 expression was invariable among castes, while most of the rest of the paralogs

822 showed caste-biased expression. (c) ML tree of GGPP synthase homologs with a

823 LG+G model. R. speratus genes are marked with blue circles. (d) Vertical

824 cryosection of the soldier head subjected to in situ hybridization for RS100016

825 mRNA. The front of the head is on the left side. The gland cell layer surrounding the

826 frontal gland reservoir $(R)$ is stained dark (arrowhead). The asterisk indicates the

827 brain. The frontal pore $(\mathrm{P})$ discharging frontal gland secretion is also observed. Bar

$828=0.1 \mathrm{~mm}$. See Supplementary Fig. $7 \mathrm{c}$ for the negative control experiment. (e)

829 Molecular evolutionary analysis of $R$. speratus GGPP synthase homologs by the

830 PAML branch-site test. Detected positive selection is marked with a single asterisk *

$831(p<0.05)$ or double asterisks ** $(p<0.01)$ next to the corresponding branches.

833 Figure 7: The TY family, a novel secretion gene family identified from termite 834 taxonomically restricted genes.

835 (a) Genomic locations and caste-biased expression patterns of TY family genes. (b)

836 Multiple alignment of TY homologs of $R$. speratus and $Z$. nevadensis. Protein motifs

837 and structural characteristics are represented. (c) Orthology of TY homologs in

838 three termites and the results of the $\mathrm{Ka} / \mathrm{Ks}$ analysis.

839

840 


\section{References}

842 1. J. M. Smith, E. Szathmary, The Major Transitions in Evolution (OUP Oxford, 843 1997).

844 2. E. O. Wilson, Others, The insect societies. The insect societies. (1971).

845 3. G. E. Robinson, C. M. Grozinger, C. W. Whitfield, Sociogenomics: social life in $846 \quad$ molecular terms. Nat. Rev. Genet. 6, 257-270 (2005).

847 4. C. M. Grozinger, Y. Fan, S. E. R. Hoover, M. L. Winston, Genome-wide 848 analysis reveals differences in brain gene expression patterns associated with caste and reproductive status in honey bees (Apis mellifera). Molecular Ecology 16, 4837-4848 (2007).

5. R. Bonasio, et al., Genomic comparison of the ants Camponotus floridanus and Harpegnathos saltator. Science 329, 1068-1071 (2010).

6. P. G. Ferreira, et al., Transcriptome analyses of primitively eusocial wasps reveal novel insights into the evolution of sociality and the origin of alternative phenotypes. Genome Biol. 14, R20 (2013).

7. R. Bonasio, et al., Genome-wide and caste-specific DNA methylomes of the ants Camponotus floridanus and Harpegnathos saltator. Curr. Biol. 22, 17551764 (2012).

8. B. Feldmeyer, D. Elsner, S. Foitzik, Gene expression patterns associated with caste and reproductive status in ants: worker-specific genes are more derived than queen-specific ones. Mol. Ecol. 23, 151-161 (2014).

9. S. Patalano, et al., Molecular signatures of plastic phenotypes in two eusocial insect species with simple societies. Proceedings of the National Academy of Sciences 112, 201515937-201513975 (2015).

10. R. Libbrecht, P. R. Oxley, L. Keller, D. J. C. Kronauer, Robust DNA Methylation in the Clonal Raider Ant Brain. Curr. Biol. 26, 391-395 (2016).

11. D. S. Standage, et al., Genome, transcriptome and methylome sequencing of a primitively eusocial wasp reveal a greatly reduced DNA methylation system in a social insect. Mol. Ecol. 25, 1769-1784 (2016).

12. D. F. Simola, et al., Epigenetic (re)programming of caste-specific behavior in the ant Camponotus floridanus. Science 351, aac6633 (2016).

13. K. M. Glastad, B. G. Hunt, M. A. D. Goodisman, DNA methylation and chromatin organization in insects: insights from the Ant Camponotus floridanus. Genome Biol. Evol. 7, 931-942 (2015).

14. S. M. Rehan, A. L. Toth, Climbing the social ladder: the molecular evolution of sociality. Trends Ecol. Evol. 30, 426-433 (2015). 
15. A. L. Toth, S. M. Rehan, Molecular Evolution of Insect Sociality: An Eco-EvoDevo Perspective. Annu. Rev. Entomol. 62, 419-442 (2017).

16. Y. Roisin, J. Korb, "Social Organisation and the Status of Workers in Termites" in Biology of Termites: A Modern Synthesis, D. E. Bignell, Y. Roisin, N. Lo, Eds. (Springer Netherlands, 2011), pp. 133-164.

17. N. Terrapon, et al., Molecular traces of alternative social organization in a termite genome. Nat. Commun. 5, 3636 (2014).

18. M. C. Harrison, et al., Hemimetabolous genomes reveal molecular basis of termite eusociality. Nat Ecol Evol 2, 557-566 (2018).

19. K. M. Glastad, K. Gokhale, J. Liebig, M. A. D. Goodisman, The caste- and sexspecific DNA methylome of the termite Zootermopsis nevadensis. Sci. Rep. 6, 37110 (2016).

20. K. Krishna, D. A. Grimaldi, V. Krishna, M. S. Engel, Treatise on the Isoptera of the world.(Bulletin of the American Museum of Natural History, no. 377) (2013).

21. D. J. G. Inward, A. P. Vogler, P. Eggleton, A comprehensive phylogenetic analysis of termites (Isoptera) illuminates key aspects of their evolutionary biology. Mol. Phylogenet. Evol. 44, 953-967 (2007).

22. T. Bourguignon, et al., The evolutionary history of termites as inferred from 66 mitochondrial genomes. Mol. Biol. Evol. 32, 406-421 (2014).

23. E. L. Vargo, C. Husseneder, Biology of subterranean termites: insights from molecular studies of Reticulitermes and Coptotermes. Annu. Rev. Entomol. 54, 379-403 (2009).

24. HARRIS, WV, Termites of the Palearctic Region. Biology of Termites, 295-313 (1970).

25. WEESNER, F. M, Termites of the nearctic region. The Biology of Termites, 477-522 (1970).

26. S. Govorushko, Economic and ecological importance of termites: A global review. Entomol. Sci. 22, 21-35 (2019).

27. K. Matsuura, et al., Queen Succession Through Asexual Reproduction in Termites. Science 323, 1687-1687 (2009).

28. S. Koshikawa, S. Miyazaki, R. Cornette, T. Matsumoto, T. Miura, Genome size of termites (Insecta, Dictyoptera, Isoptera) and wood roaches (Insecta, Dictyoptera, Cryptocercidae). Naturwissenschaften 95, 859-867 (2008).

29. M. Seppey, M. Manni, E. M. Zdobnov, BUSCO: Assessing Genome Assembly and Annotation Completeness. Methods Mol. Biol. 1962, 227-245 (2019).

30. M. Poulsen, et al., Complementary symbiont contributions to plant 
decomposition in a fungus-farming termite. Proc. Natl. Acad. Sci. U. S. A. 111, 14500-14505 (2014).

31. T. Miura, Developmental regulation of caste-specific characters in social-insect polyphenism. Evol. Dev. 7, 122-129 (2005).

32. H. F. Nijhout, Development and evolution of adaptive polyphenisms. Evol. Dev. 5, 9-18 (2003).

33. T. Wu, G. K. Dhami, G. J. Thompson, Soldier-biased gene expression in a subterranean termite implies functional specialization of the defensive caste. Evol. Dev. 20, 3-16 (2018).

34. M. Hojo, S. Shigenobu, K. Maekawa, T. Miura, G. Tokuda, Duplication and soldier-specific expression of geranylgeranyl diphosphate synthase genes in a nasute termite Nasutitermes takasagoensis. Insect Biochem. Mol. Biol. 111, 103177 (2019).

35. M. E. Scharf, D. Wu-Scharf, B. R. Pittendrigh, G. W. Bennett, Caste- and development-associated gene expression in a lower termite. Genome Biol. 4, R62 (2003).

36. M. M. Steller, S. Kambhampati, D. Caragea, Comparative analysis of expressed sequence tags from three castes and two life stages of the termite Reticulitermes flavipes. BMC Genomics 11, 463 (2010).

37. T. Weil, M. Rehli, J. Korb, Molecular basis for the reproductive division of labour in a lower termite. BMC Genomics 8, 198 (2007).

38. S. Ohno, Evolution by Gene Duplication (Springer, Berlin, Heidelberg, 1970).

39. H. Innan, F. Kondrashov, The evolution of gene duplications: classifying and distinguishing between models. Nat. Rev. Genet. 11, 97-108 (2010).

40. D. F. Simola, et al., A chromatin link to caste identity in the carpenter ant Camponotus floridanus. Genome Res. 23, 486-496 (2013).

41. K. M. Kapheim, et al., Social evolution. Genomic signatures of evolutionary transitions from solitary to group living. Science 348, 1139-1143 (2015).

42. D. R. Flower, The lipocalin protein family: structure and function. Biochem. J 318 ( Pt 1), 1-14 (1996).

43. T. Miura, et al., Soldier caste-specific gene expression in the mandibular glands of Hodotermopsis japonica (Isoptera: termopsidae). Proc. Natl. Acad. Sci. U. S. A. 96, 13874-13879 (1999).

950 45. M. Ruiz, D. Sanchez, C. Correnti, R. K. Strong, M. D. Ganfornina, Lipid-binding 
properties of human ApoD and Lazarillo-related lipocalins: functional implications for cell differentiation. FEBS J. 280, 3928-3943 (2013).

46. Y. Mitaka, et al., Caste-Specific and Sex-Specific Expression of

49. P. Pelosi, J.-J. Zhou, L. P. Ban, M. Calvello, Soluble proteins in insect chemical communication. Cell. Mol. Life Sci. 63, 1658-1676 (2006).

50. H. Watanabe, G. Tokuda, Cellulolytic Systems in Insects. Annu. Rev. Entomol. 55, 609-632 (2009).

51. G. Tokuda, Plant cell wall degradation in insects: Recent progress on endogenous enzymes revealed by multi-omics technologies. Adv. In Insect Phys. 57, 97 (2019).

52. J. Ni, G. Tokuda, Lignocellulose-degrading enzymes from termites and their symbiotic microbiota. Biotechnol. Adv. 31, 838-850 (2013).

53. K. Matsuura, T. Yashiro, K. Shimizu, S. Tatsumi, T. Tamura, Cuckoo Fungus Mimics Termite Eggs by Producing the Cellulose-Digesting Enzyme $\beta$ Glucosidase. Curr. Biol. 19, 30-36 (2009).

54. J. Korb, T. Weil, K. Hoffmann, K. R. Foster, M. Rehli, A gene necessary for reproductive suppression in termites. Science 324, 758 (2009).

55. M. Shelomi, B. Wipfler, X. Zhou, Y. Pauchet, Multifunctional cellulase enzymes are ancestral in Polyneoptera. Insect Mol. Biol. 29, 124-135 (2020).

56. Q. Gao, G. J. Thompson, Social context affects immune gene expression in a subterranean termite. Insectes Soc. 62, 167-170 (2015).

57. L. Callewaert, C. W. Michiels, Lysozymes in the animal kingdom. J. Biosci. 35 , 127-160 (2010).

58. A. Fujita, I. Shimizu, T. Abe, Distribution of lysozyme and protease, and amino acid concentration in the guts of a wood-feeding termite, Reticulitermes speratus (Kolbe): possible digestion of symbiont bacteria transferred by trophallaxis. Physiological Entomology 26, 116-123 (2001).

987 60. K. Ogura, T. Koyama, Enzymatic Aspects of Isoprenoid Chain Elongation. 
Chem. Rev. 98, 1263-1276 (1998).

989

990

991

1003

1004

1005

1006

1007

1008

1009

1010

1011

1012

1013

1014

1015

1016

1017

1018

1019

1020

1021

1022

1023

1024

1025

61. K. C. Wang, S. Ohnuma, Isoprenyl diphosphate synthases. Biochim. Biophys. Acta 1529, 33-48 (2000).

62. M. Hojo, T. Matsumoto, T. Miura, Cloning and expression of a geranylgeranyl diphosphate synthase gene: insights into the synthesis of termite defence secretion. Insect Mol. Biol. 16, 121-131 (2007).

63. G. D. Prestwich, The chemicals of termite societies (Isoptera). Sociobiology 14, 175-191 (1988).

64. L. J. Nelson, L. G. Cool, B. T. Forschler, M. I. Haverty, Correspondence of Soldier Defense Secretion Mixtures with Cuticular Hydrocarbon Phenotypes for Chemotaxonomy of the Termite Genus Reticulitermes in North America. J. Chem. Ecol. 27, 1449-1479 (2001).

65. A. Quintana, et al., Interspecific variation in terpenoid composition of defensive secretions of European Reticulitermes termites. J. Chem. Ecol. 29, 639-652 (2003).

66. M. Hojo, K. Toga, D. Watanabe, T. Yamamoto, K. Maekawa, High-level expression of the Geranylgeranyl diphosphate synthase gene in the frontal gland of soldiers in Reticulitermes speratus (Isoptera: Rhinotermitidae). Arch. Insect Biochem. Physiol. 77, 17-31 (2011).

67. Z. Yang, R. Nielsen, Codon-substitution models for detecting molecular adaptation at individual sites along specific lineages. Mol. Biol. Evol. 19, 908917 (2002).

68. B. R. Johnson, N. D. Tsutsui, Taxonomically restricted genes are associated with the evolution of sociality in the honey bee. BMC Genomics 12, 164 (2011).

69. S. Sumner, The importance of genomic novelty in social evolution. Mol. Ecol. 23, 26-28 (2014).

70. C. R. Smith, et al., Genetic and genomic analyses of the division of labour in insect societies. Nat. Rev. Genet. 9, 735-748 (2008).

71. M. Lynch, J. S. Conery, The evolutionary fate and consequences of duplicate genes. Science 290, 1151-1155 (2000).

72. M. Long, E. Betrán, K. Thornton, W. Wang, The origin of new genes: glimpses from the young and old. Nat. Rev. Genet. 4, 865-875 (2003).

73. G. C. Conant, K. H. Wolfe, Probabilistic cross-species inference of orthologous genomic regions created by whole-genome duplication in yeast. Genetics 179, 1681-1692 (2008).

74. R. Gadagkar, The evolution of caste polymorphism in social insects:0 Genetic release followed by diversifying evolution. Journal of Genetics 76, 167-179 
(1997).

75. S. K. McKenzie, I. Fetter-Pruneda, V. Ruta, D. J. C. Kronauer, Transcriptomics and neuroanatomy of the clonal raider ant implicate an expanded clade of odorant receptors in chemical communication. Proceedings of the National Academy of Sciences 113, 14091-14096 (2016).

76. X. Zhou, et al., Phylogenetic and transcriptomic analysis of chemosensory receptors in a pair of divergent ant species reveals sex-specific signatures of odor coding. PLoS Genet. 8, e1002930 (2012).

77. X. Zhou, et al., Chemoreceptor Evolution in Hymenoptera and Its Implications for the Evolution of Eusociality. Genome Biol. Evol. 7, 2407-2416 (2015).

78. L. M. Chau, M. A. D. Goodisman, Gene duplication and the evolution of phenotypic diversity in insect societies. Evolution 71, 2871-2884 (2017).

79. K. Maekawa, K. Ishitani, H. Gotoh, R. Cornette, T. Miura, Juvenile Hormone titre and vitellogenin gene expression related to ovarian development in primary reproductives compared with nymphs and nymphoid reproductives of the termiteReticulitermes speratus. Physiological Entomology 35, 52-58 (2010).

80. A. Bucek, et al., Evolution of Termite Symbiosis Informed by TranscriptomeBased Phylogenies. Curr. Biol. 29, 3728-3734.e4 (2019). 
bioRxiv preprint doi: https://doi.org/10.1101/2021.07.11.451559; this version posted July 12, 2021. The copyright holder for this preprint (which was not certified by peer review) is the author/funder. All rights reserved. No reuse allowed without permission.

Fig. 1

a
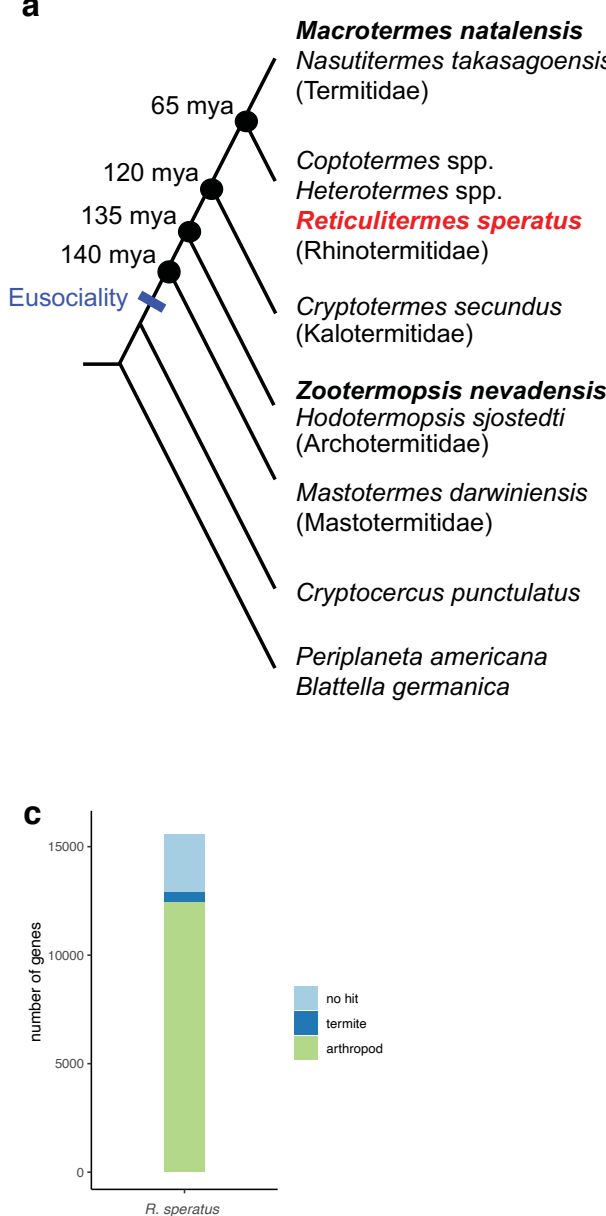

b

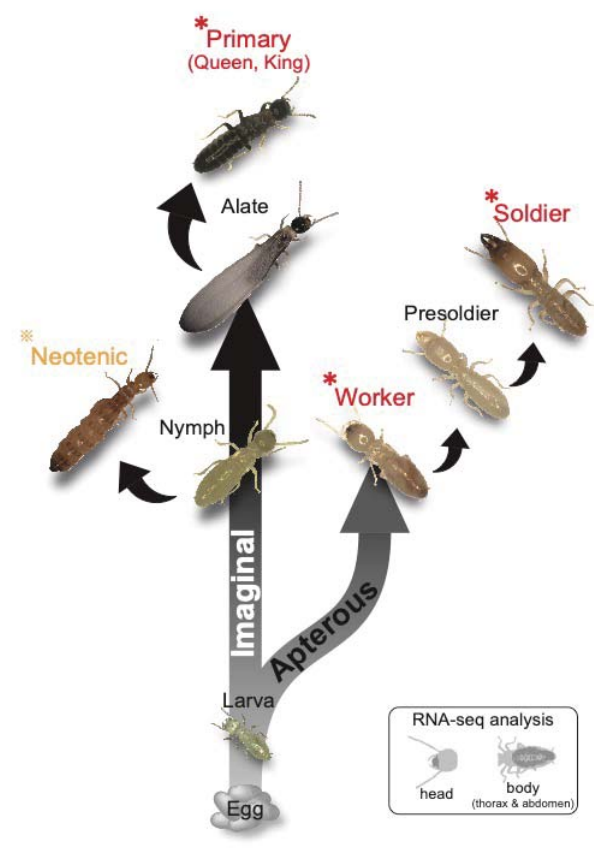

d

M. natalensis

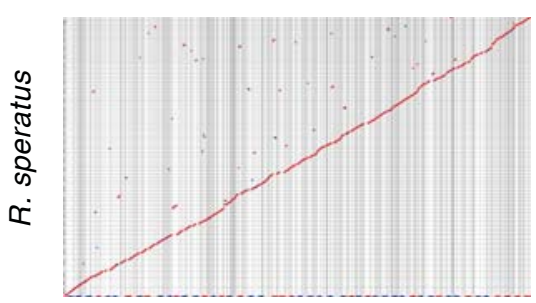

Z. nevadensis

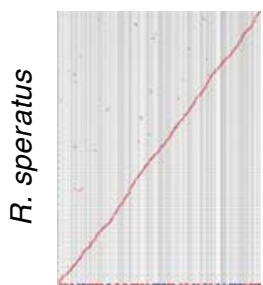


bioRxiv preprint doi: https://doi.org/10.1101/2021.07.11.451559; this version posted July 12, 2021. The copyright holder for this preprint (which was not certified by peer review) is the author/funder. All rights reserved. No reuse allowed without permission.

Fig. 2

a
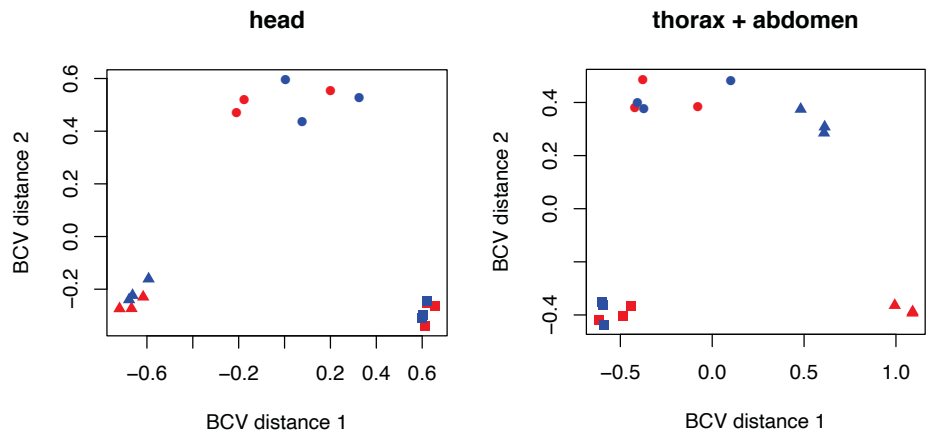

$\Delta:$ reproductive

口: soldier

: worker

Red: female

Blue: male

b

c
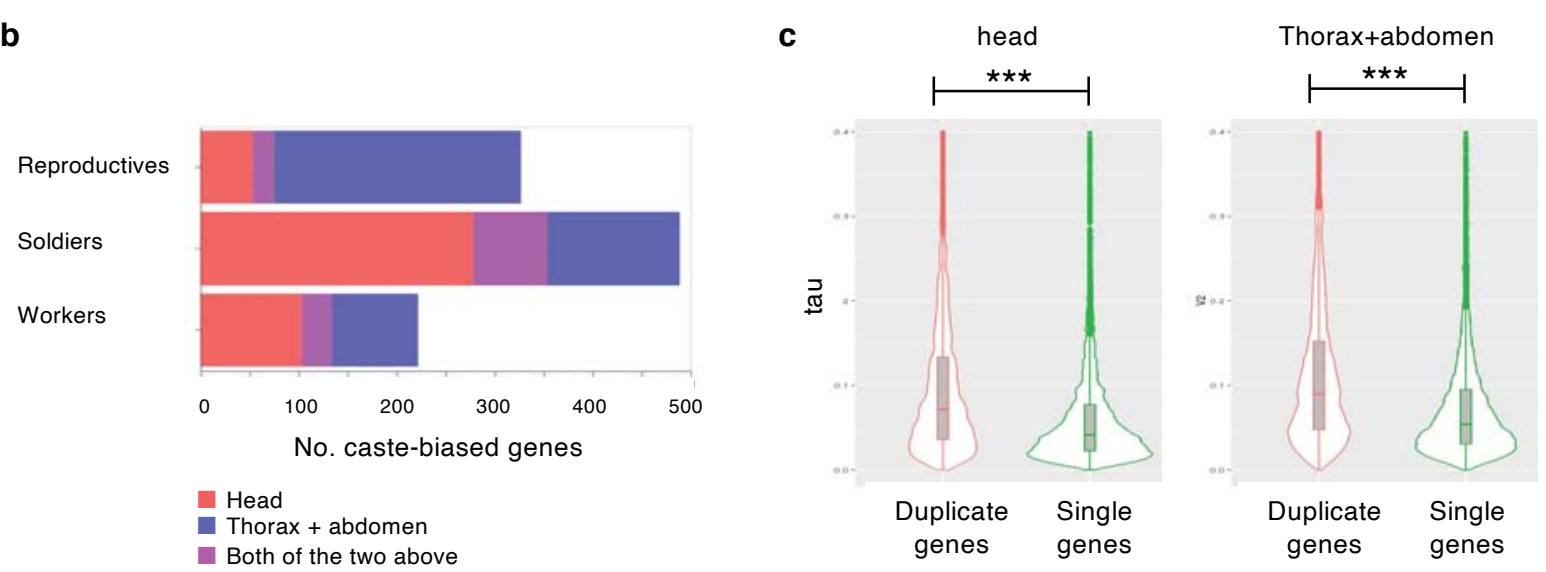

\section{d}
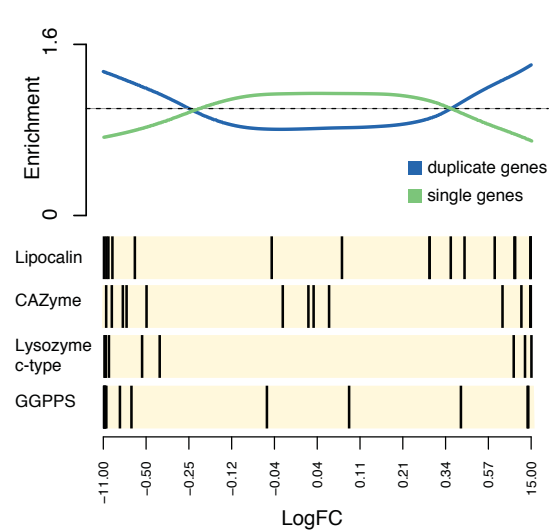

Reproductive vs Worker
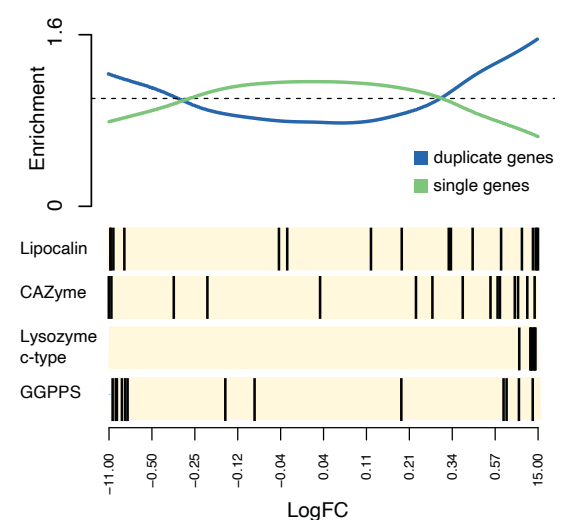

Reproductive vs Soldier

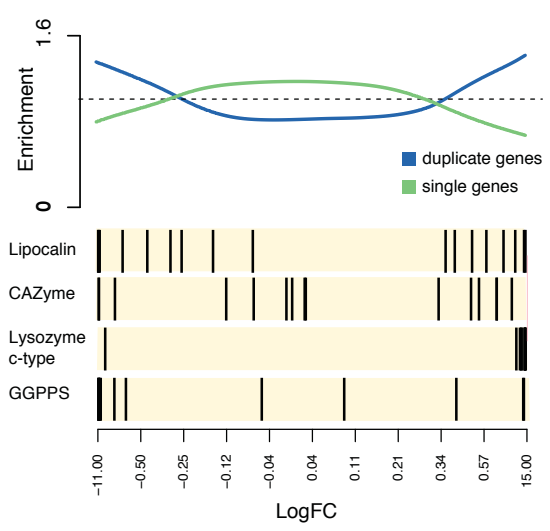


bioRxiv preprint doi: https://doi.org/10.1101/2021.07.11.451559; this version posted July 12, 2021. The copyright holder for this preprint (which was not certified by peer review) is the author/funder. All rights reserved. No reuse allowed without permission.

Fig. 3

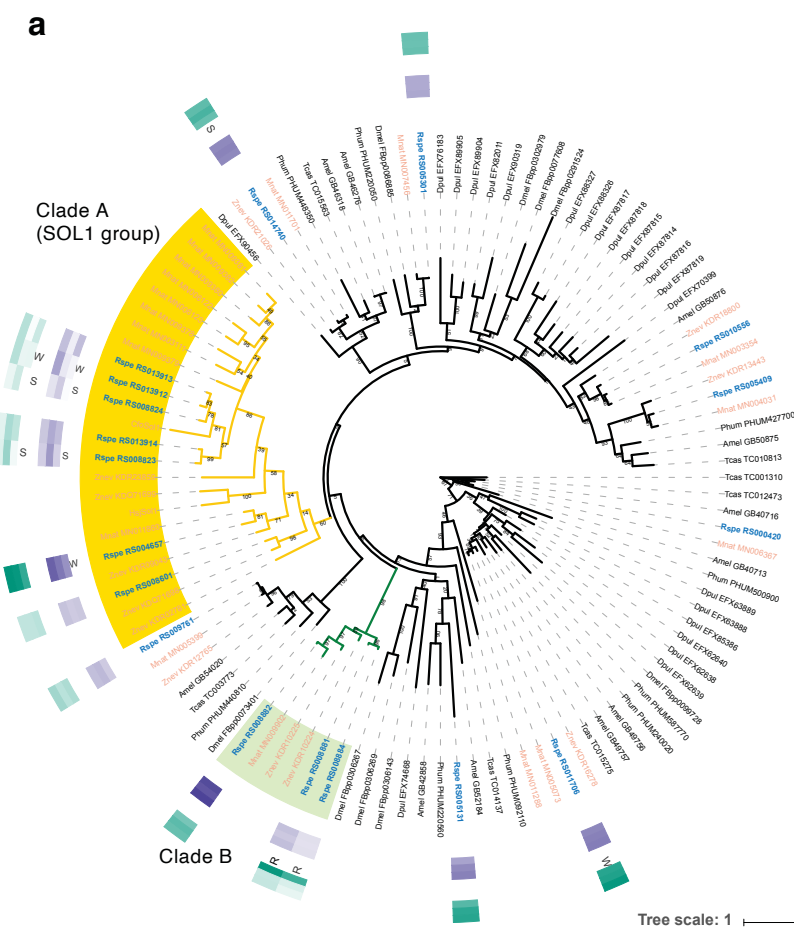

b
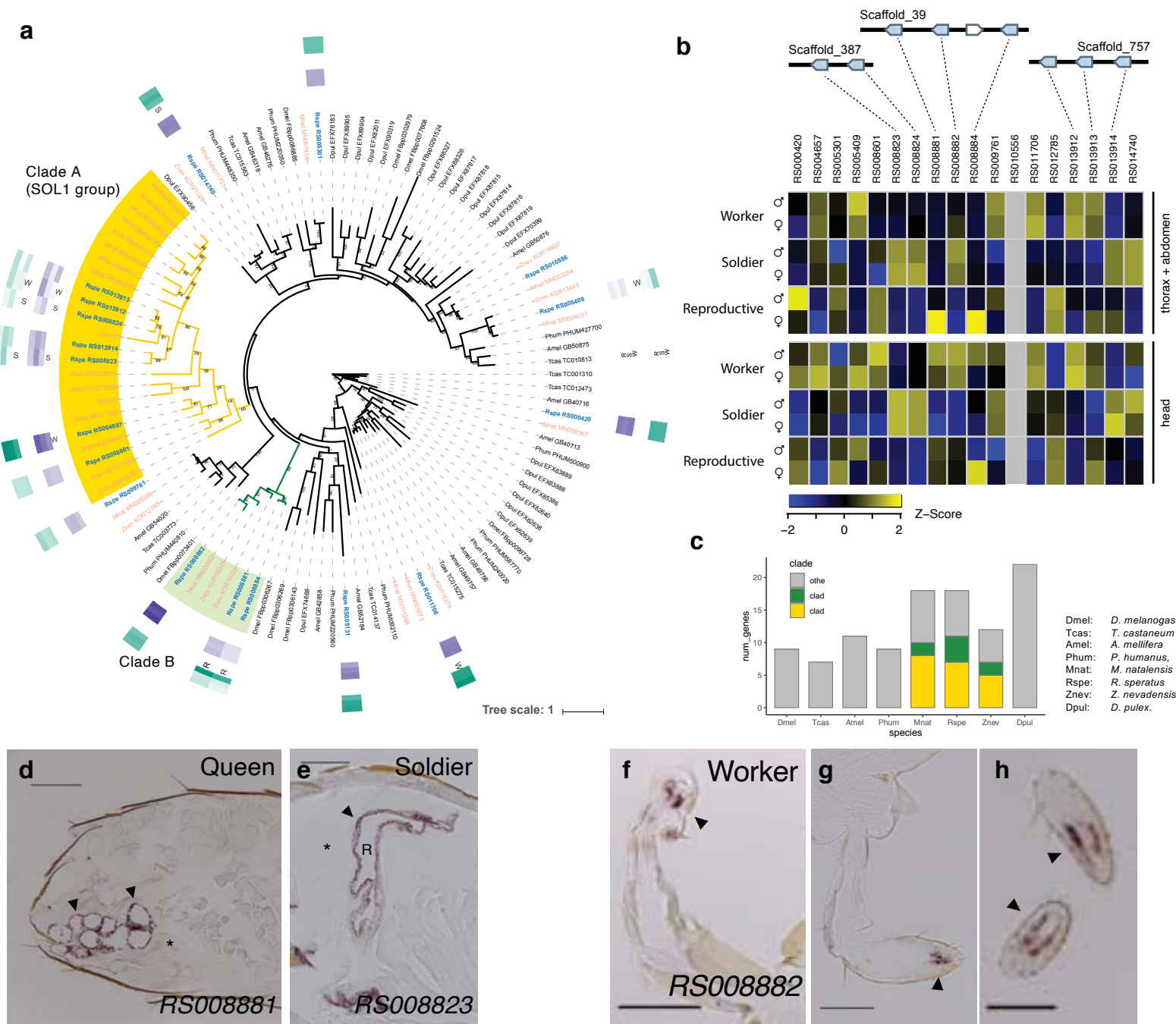
bioRxiv preprint doi: https://doi.org/10.1101/2021.07.11.451559; this version posted July 12, 2021. The copyright holder for this preprint (which was not certified by peer review) is the author/funder. All rights reserved. No reuse allowed without permission.

Fig. 4

a

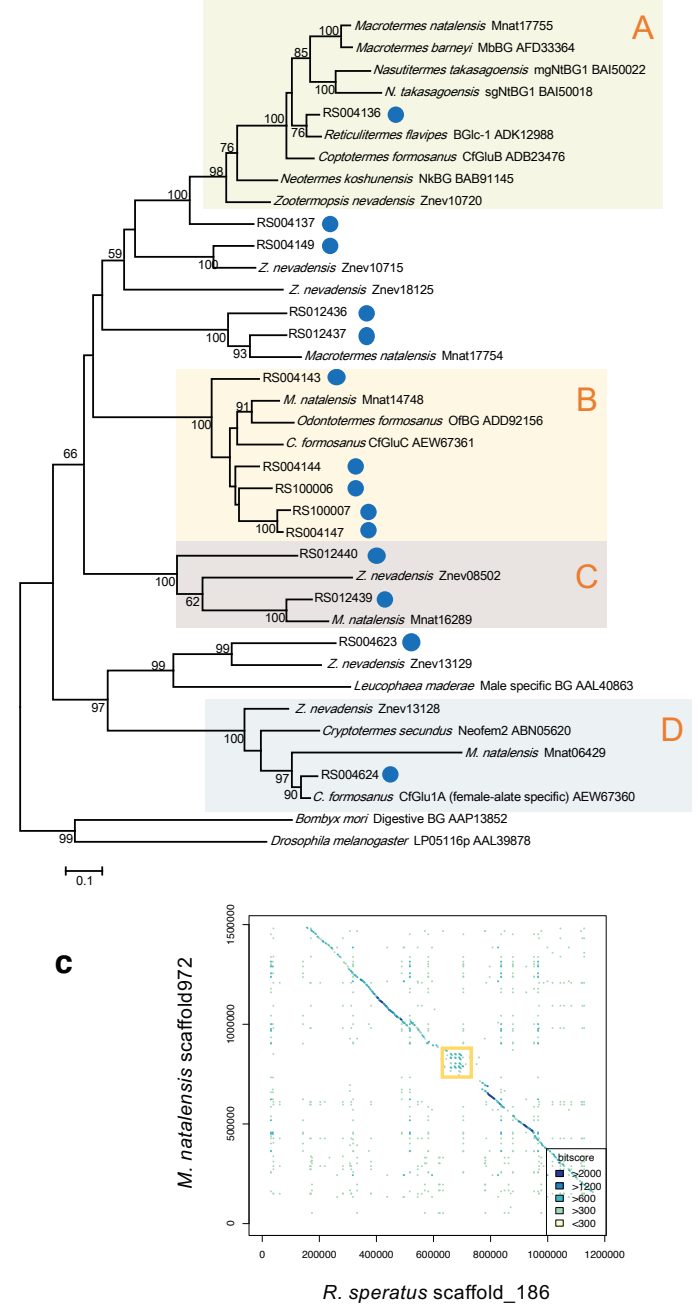

b
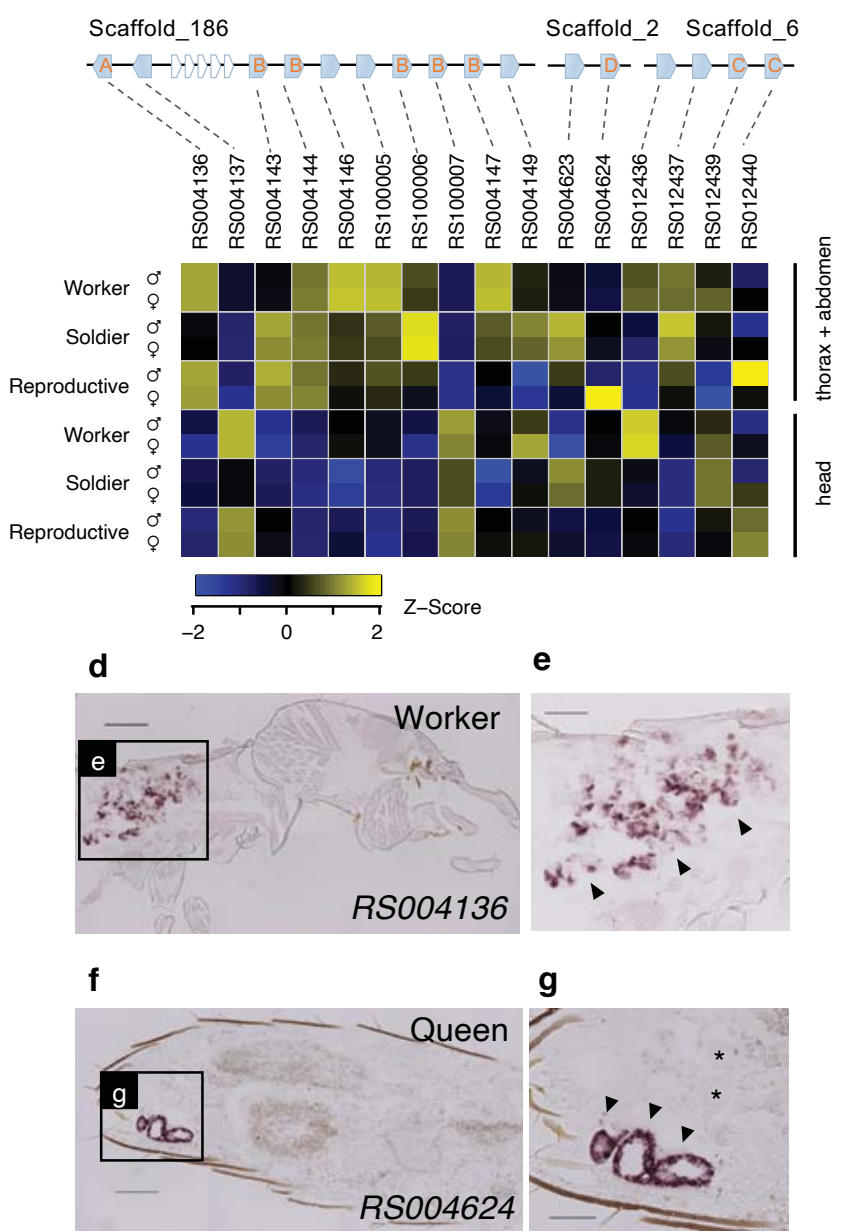

g

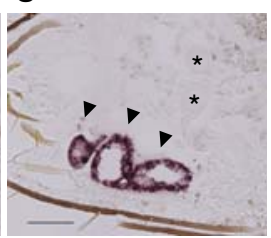


bioRxiv preprint doi: https://doi.org/10.1101/2021.07.11.451559; this version posted July 12, 2021. The copyright holder for this preprint (which was not certified by peer review) is the author/funder. All rights reserved. No reuse allowed without permission.

Fig. 5

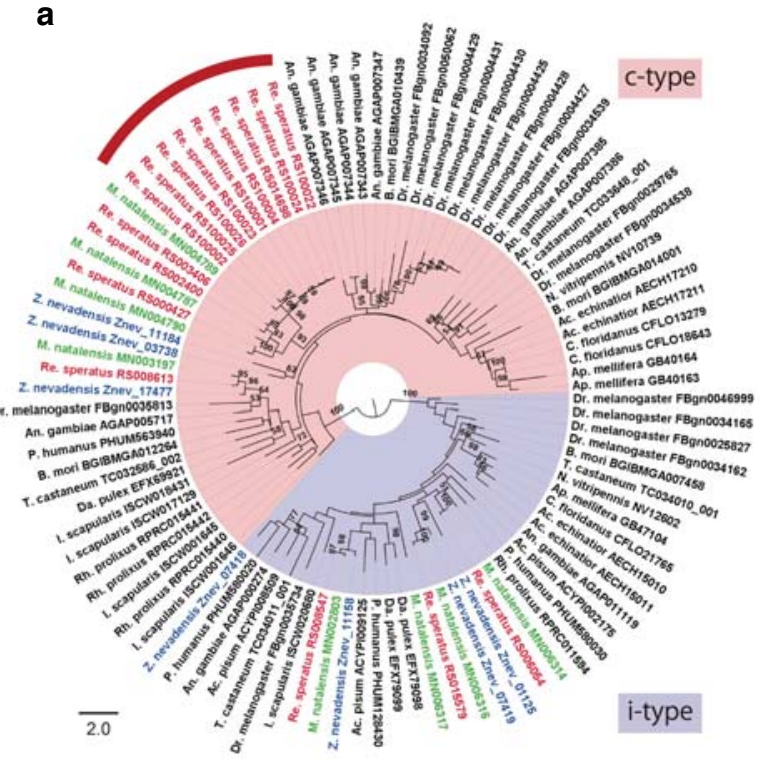

b

Scaffold_1097 Scaffold_859
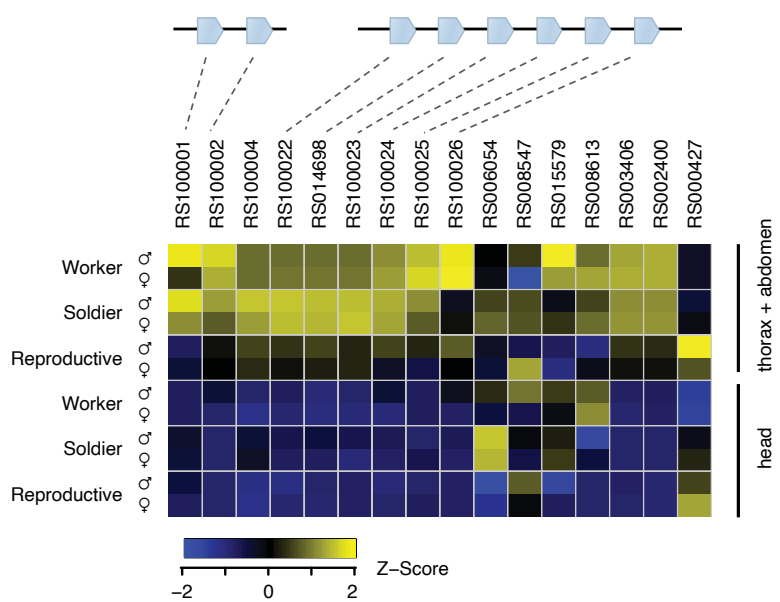
bioRxiv preprint doi: https://doi.org/10.1101/2021.07.11.451559; this version posted July 12, 2021. The copyright holder for this preprint (which was not certified by peer review) is the author/funder. All rights reserved. No reuse allowed without permission.

Fig. 6

a

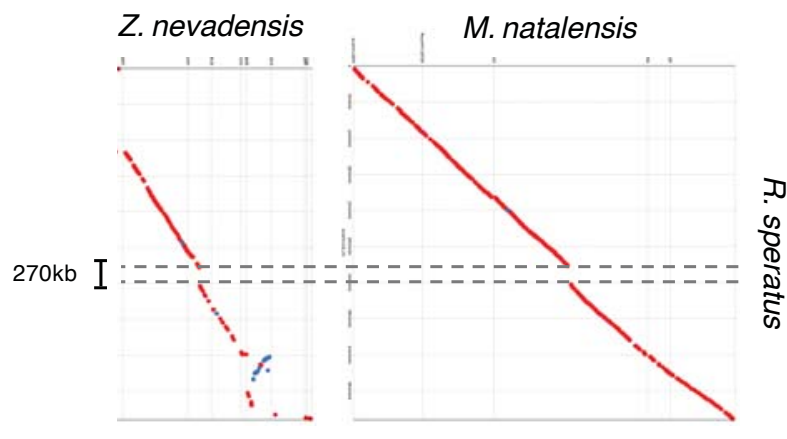

b

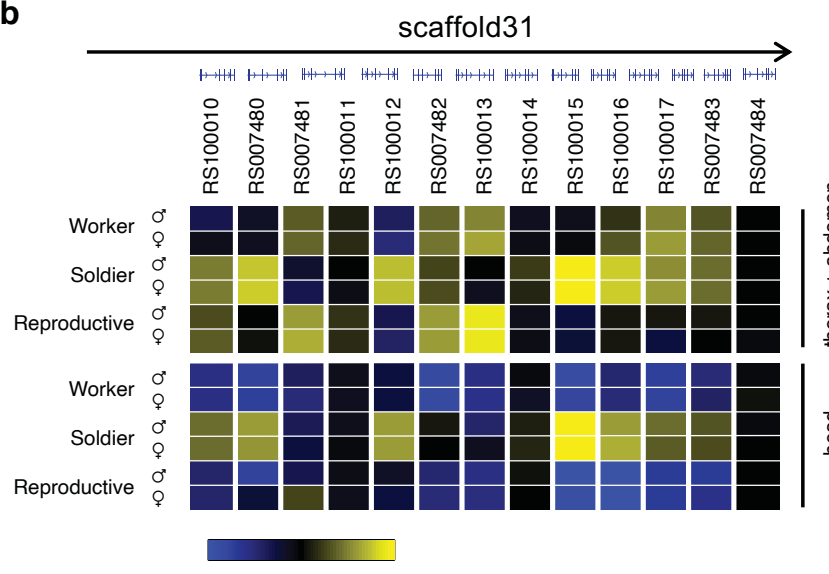

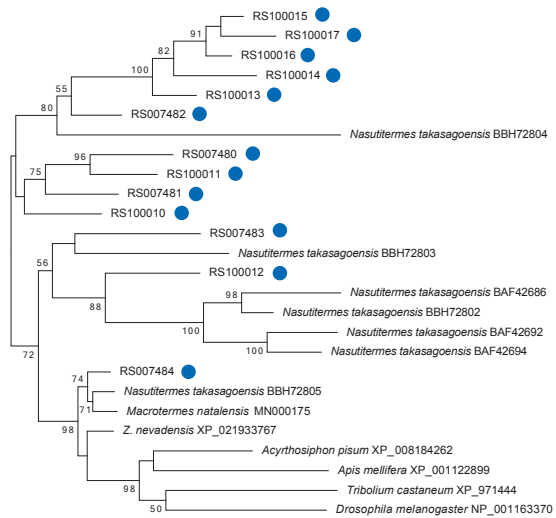

d
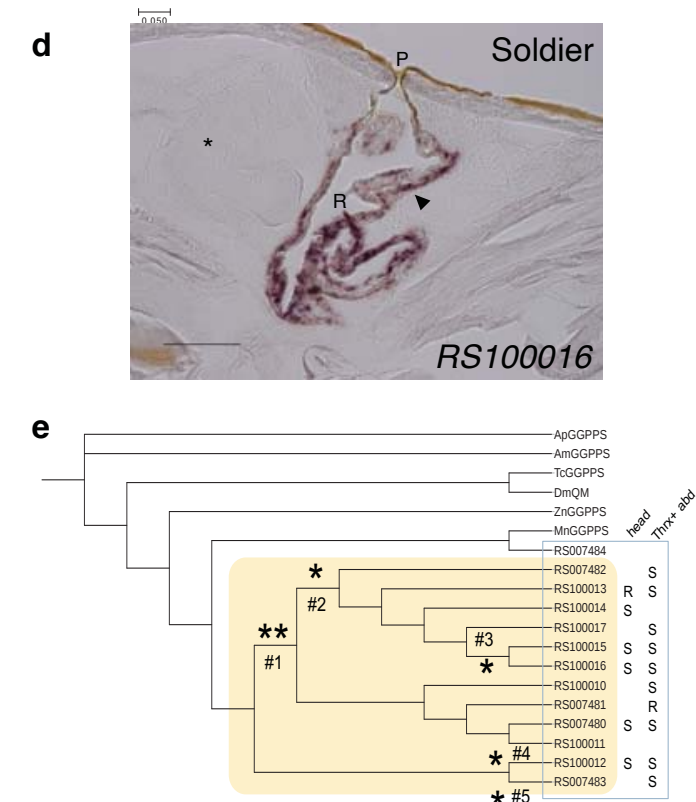
bioRxiv preprint doi: https://doi.org/10.1101/2021.07.11.451559; this version posted July 12, 2021. The copyright holder for this preprint (which was not certified by peer review) is the author/funder. All rights reserved. No reuse allowed without permission.

Fig. 7

a
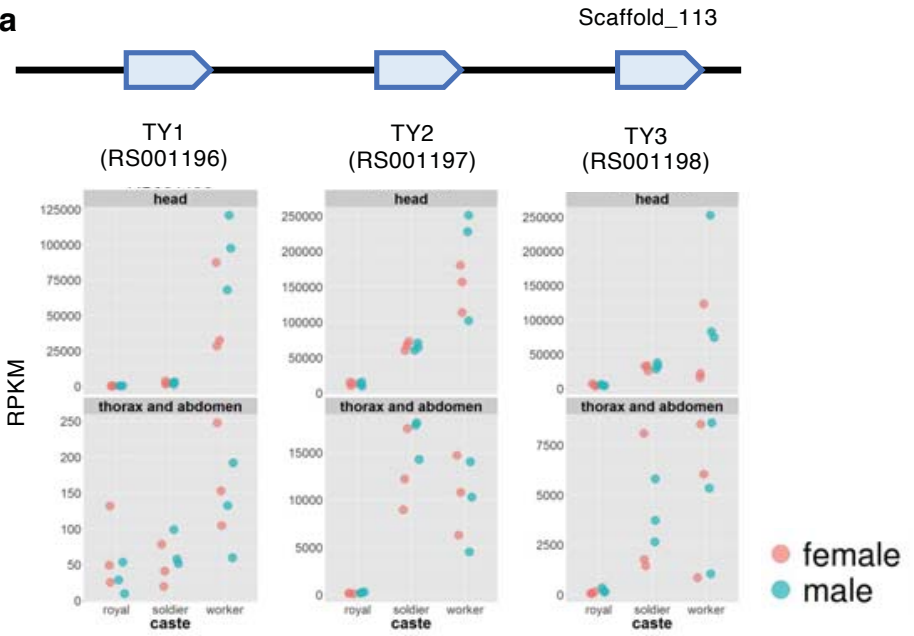

b

b Secretion signal

Highly charged

Highly polar / Tyr rich

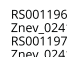

MNK

c

\begin{tabular}{cccc}
\hline & orthology & & Ka/Ks \\
R. speratus & Z. nevadensis & M. natalensis & Rspe vs Znev \\
\hline RS001196 & Znev_02416 & MN011308 & 0.147 \\
RS001197 & Znev_02414 & & 0.162 \\
RS001198 & Znev_02413 & MN011309 & 0.033 \\
\hline
\end{tabular}

